1 Dmp1Cre-directed knockdown of PTHrP in murine decidua is associated with increased bone width and a life-long increase in strength specific to male progeny

3 Niloufar Ansari1,2,3, Tsuyoshi Isojima ${ }^{1,4}$, Blessing Crimeen-Irwin ${ }^{1}$, Ingrid J Poulton ${ }^{1}$, 4 Narelle E. McGregor ${ }^{1}$, Patricia W. M. Ho ${ }^{1}$, Christopher S Kovacs ${ }^{5}$, Evdokia Dimitriadis ${ }^{6}$, 5 Jonathan H Gooi ${ }^{1,2,7}$, T. John Martin ${ }^{1,2}$, Natalie A. Sims ${ }^{1,2, *}$

$6 \quad{ }^{1}$ St. Vincent's Institute of Medical Research, Fitzroy, Victoria, Australia

72 The University of Melbourne, Department of Medicine at St. Vincent's Hospital, Fitzroy,

8 Victoria, Australia

$9{ }^{3}$ Current address: Drug Delivery, Disposition and Dynamics, Monash Institute of

10 Pharmaceutical Sciences, Monash University, Parkville, Victoria, Australia

114 Teikyo University School of Medicine, Department of Pediatrics, Tokyo, Japan

125 Memorial University of Newfoundland, St John's, Newfoundland, Canada

13 'Department of Obstetrics and Gynecology, University of Melbourne, The Women's 14 Hospital, Melbourne, Australia

$15{ }^{7}$ Bio21 Molecular Science and Biotechnology Institute, Parkville, Victoria, Australia

16 Corresponding author:

17 Natalie A Sims

189 Princes St

19 Fitzroy, Victoria 3122

20 Australia

21 Email: nsims@svi.edu.au

22 Phone: $+613-9231-2555$

23 Fax: $+613-9416-2676$

\title{
24 Abstract
}

Parathyroid hormone related-protein (PTHrP) is a pleiotropic regulator of tissue homeostasis. In bone, knockdown in osteocytes by Dmp1Cre-targeted deletion causes osteopenia and impaired strength. We report that this outcome depends on parental genotype. Adult Dmp1Cre.Pthlhf/f mice from homozygous parents (Dmp1Cre.Pthlhf/f(hom)) have stronger bones, with $40 \%$ more trabecular bone mass and 30\% greater femoral width than controls. At 12 days old, greater bone width was also found in male and female Dmp1Cre.Pthlhf/f(hom) mice, but not in gene-matched mice from heterozygous parents, suggesting a maternal influence before weaning. Milk PTHrP levels were normal, but decidua from mothers of Dmp1Cre.Pthlhfff(hom) mice were smaller, with low PTHrP levels. Moreover, Dmp1Cre.Pthlhf/f(hom) embryonic bone was more mineralized and wider than control. We conclude that Dmp1Cre leads to gene recombination in decidua, and that decidual PTHrP influences decidual cell maturation and limits embryonic bone growth. This identifies a maternal-derived developmental origin of adult bone strength. 
Bone size and geometry are among the many factors determining bone strength (1). During skeletal development, bone grows in both the longitudinal and radial axes. Longitudinal growth is mediated by chondrocytes at the growth plates, where hypertrophic chondrocytes cease dividing, enlarge and eventually mineralize surrounding matrix (2). Simultaneously, expansion of bone diameter (termed "radial growth") balances bone length and width. Although longitudinal growth has been studied widely, little is known about the signaling pathways orchestrating radial growth (3).

Parathyroid hormone-related protein (PTHrP, gene name: Pthlh) is produced by many tissues, and acts locally to maintain their physiological function (4). While global knockout of PTHrP is neonatal lethal and causes widespread skeletal defects including reduced bone length, due largely to PTHrP's role in promoting chondrocyte maturation (5), heterozygous Pthlh deletion causes osteopenia in adult mice (6). Local PTHrP production by bone cells is also required for normal bone formation in adults during bone remodeling. This was established by studies in genetically altered mice; mice with Pthlh knockdown targeted to osteoblasts (Col1(2.3kb)Cre.Pthlhf/f) (6) or to osteocytes (Dmp1(10kb)Cre) (7) both exhibiting low bone formation and osteopenia in adulthood.

Here we report an effect of parental genotype on the bone structure of Dmp1Cre.Pthlhf/f mice. This study arose from an unexpected finding when in follow up of our previous study (7), we sought to assess the effect of Dmp1Cre-targeted knockdown of PTHrP in osteocyte in older mice. For this, we changed our breeding strategy from using heterozygous breeders to homozygous breeders to limit mouse wastage. As in previous studies from our laboratory $(8,9)$, we generated these mice using cousin-bred homozygous breeding pairs. To our surprise, adult male PTHrP-deficient mice generated from homozygous breeders (denoted Dmp1Cre.Pthlhf/f(hom) exhibited an opposing phenotype to that of mice used in our previous work, generated from heterozygous breeders (denoted Dmp1Cre.Pthlhf/f(het) (7): adult male Dmp1Cre.Pthlhf/f(hom) mice had high trabecular bone mass, and wide long bones, but normal body weight and normal bone length. Since this was a profound and reproducible phenotype, we sought to determine the parental source of the defect in bone structure.

Although we previously reported that Dmp1Cre can lead to gene recombination in the mammary gland (7), there was no alteration in milk PTHrP levels in Dmp1Cre.Pthlhf/f(het) dams. However, suckling male and female Dmp1Cre.Pthlhf/f(hom) mice both exhibited the wide bone phenotype. We traced the phenotype back to fetal development and found it was associated with low PTHrP levels in decidua basalis and impaired decidualization in mothers of Dmp1Cre.Pthlhf/f(hom) mice. This implies that PTHrP from the decidua limits bone radial growth in male and female mice, and that this has life-long effects on skeletal size in males, that override the effects of endogenous PTHrP deletion in osteocytes. This 
has significant implications for bone development, sex-differences in bone growth, and for breeding strategies used with Dmp1Cre-targeted mouse models.

\section{Results}

Adult male Dmp1Cre.Pthlhf/f(hom) mice have a high trabecular bone mass set-point, reached before 14 weeks of age

In contrast to our previous experiments showing osteopenia in 12 week old Dmp1Cre.Pthlhf/f(het) mice (7), adult male Dmp1Cre.Pthlhf/f(hom) mice (i.e. mice bred from parents expressing Dmp1Cre and homozygous for the Pthlhf/f genotype, Figure 1A) had greater trabecular bone volume than age- and sex-matched controls (Figure 1B-G). At 14, 16 and 26 weeks of age, male Dmp1Cre.Pthlhfff(hom) mice had significantly higher trabecular bone volume (Figure 1B) and trabecular number (Figure 1C) than age- and sex-matched controls. This phenotype was stable; the proportional difference in trabecular bone volume and number between genotypes was the same at all three time points assessed: i.e. trabecular bone volume and number were at a constant $\sim 140 \%$ and $\sim 133 \%$ of sex-matched male controls. Trabecular thickness remained unchanged in Dmp1Cre.Pthlhf/f(hom) mice compared to sex- and age-matched controls at all time points (Figure 1D). Trabecular separation was $\sim 18 \%$ lower than controls in male Dmp1Cre.Pthlhfff(hom) mice, and was statistically significant only at the age of 14 weeks (Figure 1E).

When representative images were generated to show this difference in trabecular bone mass (Figure 1G), we noted that male Dmp1Cre.Pthlhf/f(hom) mice also had wider bones; when measured in the trabecular region of analysis, metaphyseal periosteal perimeter was significantly higher in male Dmp1Cre.Pthlhf/f(hom) mice at all three time points (Figure $1 \mathrm{~F})$, confirming the wider bone phenotype of these mice. When Dmp1Cre.Pthlhf/f(het) mice were bred and aged to 16 and 26 weeks, no significant difference in trabecular structure or metaphyseal bone perimeter was detected at either age between Dmp1Cre.Pthlhf/f(het) mice and their controls (Figure 1 Supplement 1). These indicate that male Dmp1Cre.Pthlhf/f(hom) mice have a different phenotype to Dmp1Cre.Pthlhf/f(het) mice (7), although they have the same genotype, showing that parental genotype influences trabecular bone mass and bone radial growth.

The high trabecular bone mass in Dmp1Cre.Pthlhf/f(hom) mice was sex-specific. Female Dmp1Cre.Pthlhf/f(hom) mice showed no significant difference in trabecular bone volume, trabecular number, or metaphyseal periosteal perimeter compared to Dmp1Cre(hom) controls (Figure 1B-F). They did have lower trabecular separation at 16 weeks (Figure $1 \mathrm{E})$, implying a mild and transient elevation in trabecular bone mass. 
Figure 1

A

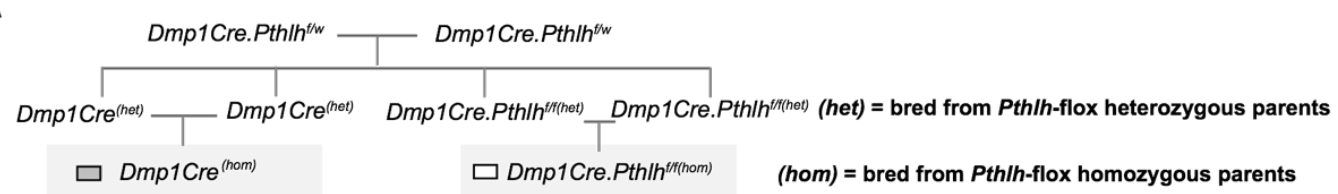

Birth 0

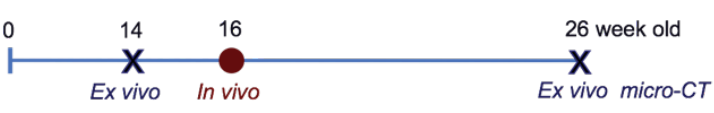

B

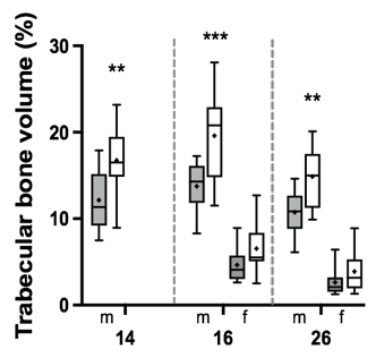

E Age (weeks)

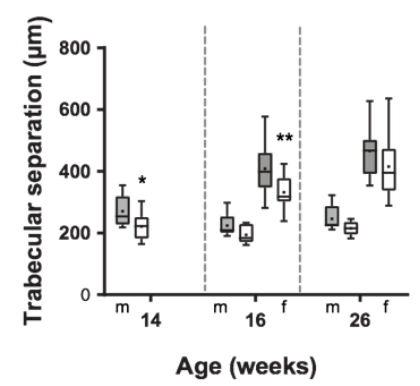

C

$\mathbf{F}$
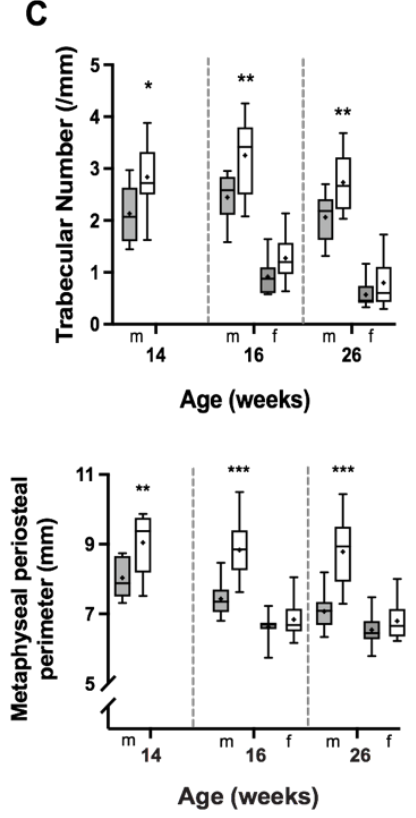

D

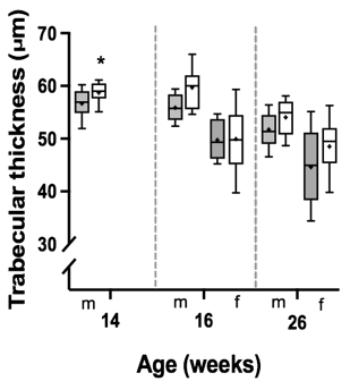

$\square \mathrm{Dmp1Cre^{( \text {mom } ) }}$

$\square$ Dmp1Cre.Pthlhithom)

G

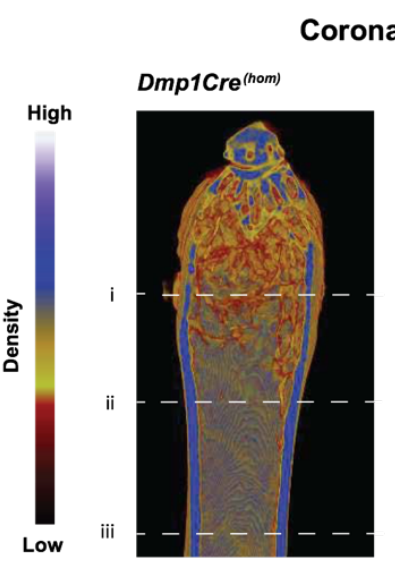

Coronal view

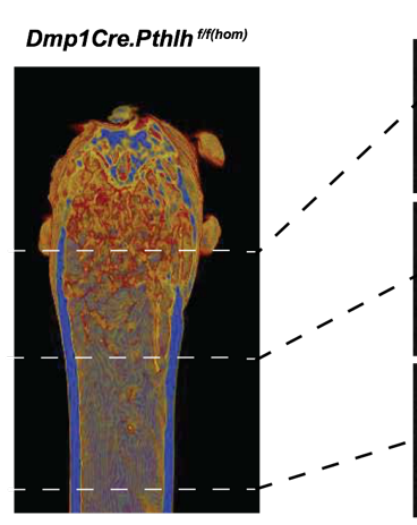

Dmp1Cre ${ }^{(\text {hom })}$

Transaxial view

112

Figure 1: Breeding strategy and high trabecular bone mass in 14, 16 and 26 week old

male Dmp1Cre.Pthlhf/f(hom) mice. A: Schematic showing breeding strategy and data collection points. B-F: Trabecular structure of Dmp1Cre.Pthlhfff(hom) distal femoral primary spongiosa analysed by micro-CT in male mice (m) at 14,16 and 26 weeks of age, and in female mice (f) at 16 and 26 weeks of age. Trabecular bone volume, trabecular number, trabecular thickness, trabecular separation, and metaphyseal periosteal perimeter are shown as mean (dot), interquartile range (box), median (line) and range; $\mathrm{n}=9-10 /$ group. ${ }^{*} \mathrm{p}<0.05,{ }^{* *} \mathrm{p}<0.01$, *** $<0.001$ compared to sex- and age-matched Dmp1Cre(hom) by two-way ANOVA (16 and 26 weeks old) and Student's t-test (14 weeks old). G: Representative micro-CT images of trabecular bone in the distal femoral primary spongiosa of 26-week old male mice, showing density (scale above images), and raw cross-sectional images of the metaphysis (i), metaphyseal diaphysis (ii) and diaphysis (iii), showing a difference in bone size, and projection of trabecular bone into the lower metaphysis in Dmp1Cre.Pthlhf/f(hom) samples. 
115 To confirm this trabecular phenotype in homozygous-bred mice, trabecular bone

116 structure was studied at a second anatomical region, $5^{\text {th }}$ lumbar (L5) vertebrae. Similar

117 to long bones, vertebrae of 14 week old male Dmp1Cre.Pthlhf/f(hom) had higher trabecular

118 bone volume and trabecular number than controls (Table 1). Trabecular separation was

119 lower in male Dmp1Cre.Pthlhf/f(hom) mice than controls, and there was no significant

120 difference in trabecular thickness (Table 1). This confirmed the trabecular phenotype

121 was not restricted to a single anatomical location.

122 Although trabecular bone mass was greater in male 14 week old Dmp1Cre.Pthlhf/f(hom)

123 mice, dynamic histomorphometry revealed no difference in any bone formation or 124 resorption parameters compared to controls (Table 2). In addition, no significant 125 difference was detected in serum levels of the bone formation or resorption markers, 126 P1NP and CTX1, of 14 week old male Dmp1Cre.Pthlhf/f(hom) mice compared to controls 127 (Table 2). This, and the similar proportion of elevation in trabecular bone mass at 14, 16 128 and 26 weeks suggest that the high trabecular bone mass arose before 14 weeks of age, 129 and has reached a greater adult set-point for "peak bone mass" than controls.

130 Adult male Dmp1Cre.Pthlhf/f(hom) mice have a wide bone phenotype, reached before 14 131 weeks of age, that leads to greater bone strength

132 Since the metaphyseal bone width was greater in adult male Dmp1Cre.Pthlhf/f(hom) mice 133 than controls, we analysed femoral cortical bone structure in more detail (Figure 2A). 134 Although femoral length was not different between Dmp1Cre.Pthlhf/f(hom) mice and

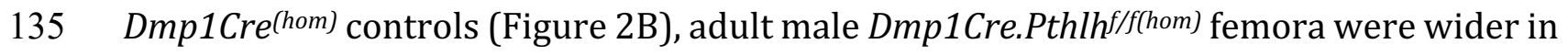
136 both anteroposterior and mediolateral dimensions compared to controls at 14, 16 and 26 137 weeks (Figure 2C,D). The greater femoral width in male Dmp1Cre.Pthlhf/f(hom) mice was in 138 proportion: the ratio of anteroposterior to mediolateral widths was not different in these 139 mice compared to controls at any time point (data not shown). Adult male 140 Dmp1Cre.Pthlhf/f(hom) mice exhibited greater marrow and cortical area, and greater 141 periosteal and endocortical perimeters, compared to age- and sex-matched controls 142 (Figure 2E-I). Although cortical diameter was greater, this was balanced on the 143 endocortical and periosteal surfaces, as there was no significant difference in cortical 144 thickness (Figure 2G). As in trabecular bone, the greater cortical bone width phenotype 145 was stable, showing a similar proportional difference compared to controls at all three 146 time points.

147 Female Dmp1Cre.Pthlhf/f(hom) mice showed no significant difference in cortical bone size 148 or shape compared to age-matched Dmp1Cre(hom) mice at 16 or 26 weeks of age (Figure 149 2).

150 Heterozygous-bred Dmp1Cre.Pthlhf/f(het) mice at 16 and 26 weeks of age did not exhibit 151 any significant difference in anteroposterior or mediolateral femoral width compared to 


\section{Figure 2}

A

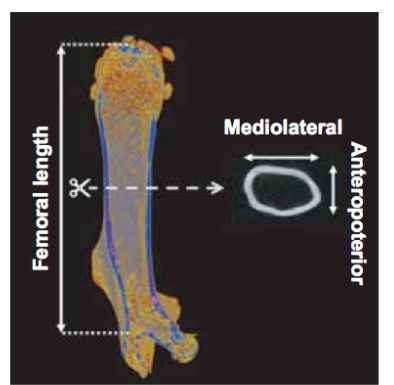

D

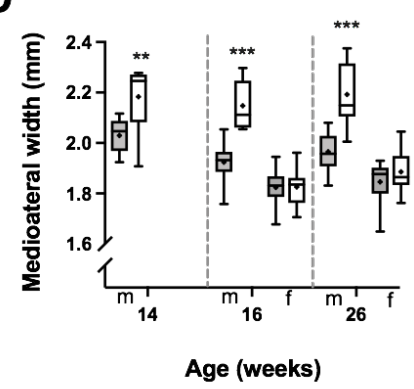

G

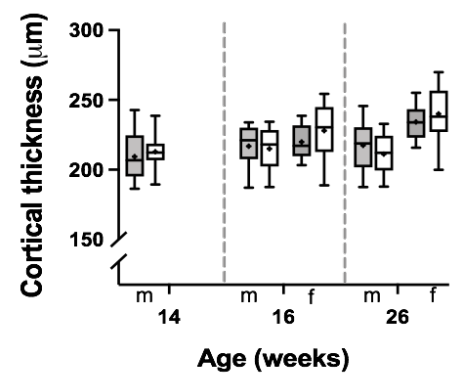

B

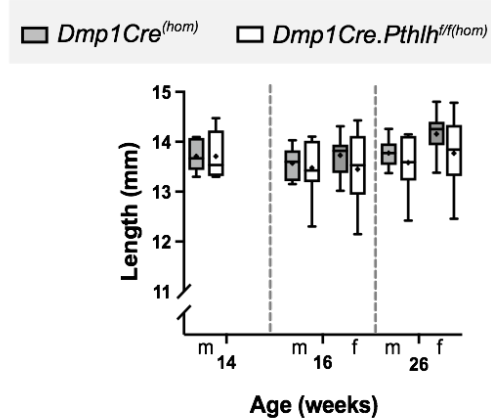

E

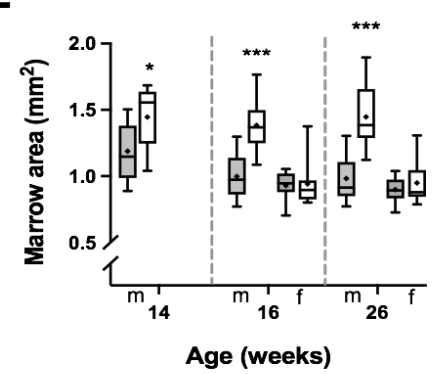

H

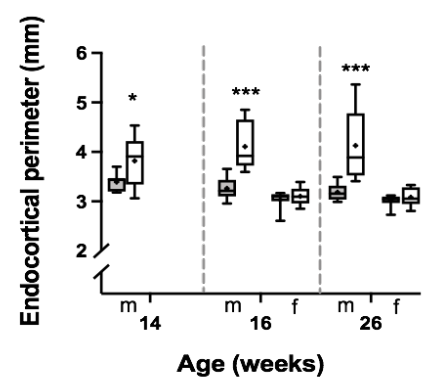

C

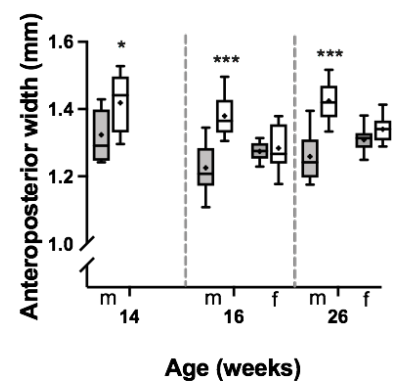

$\mathbf{F}$

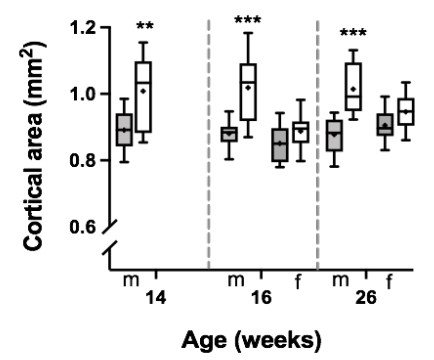

I

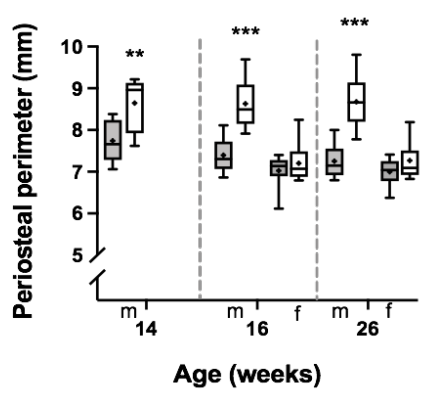
of male (m) Dmp1Cre.Pthlhf/f(hom) and Dmp1Cre(hom) mice at 14, 16 and 26 weeks of age, and female mice (f) at 16 and 26 weeks of age. Anteroposterior (C) and mediolateral (D) width, measured by micro-CT at the midshaft. E:I Femoral marrow area $(\mathbf{E})$, cortical bone area $(\mathbf{F})$, thickness $(\mathbf{G})$, and both endocortical $(\mathbf{H})$ and periosteal (I) perimeter were analysed in cortical ROI by micro-CT . Data are shown as mean (dot), interquartile range (box), median (line) and range; $n=9-10$ /group. ${ }^{*} \mathrm{p}<0.05,{ }^{* *} \mathrm{p}<0.01,{ }^{* * *} \mathrm{p}<0.001$ compared to sex- and age-matched $\mathrm{Dmp} 1 \mathrm{Cre} \mathrm{(hom)}^{(\mathrm{hy}}$ two-way ANOVA (16 and 26 weeks old) and Student's t-test (14 weeks old). 


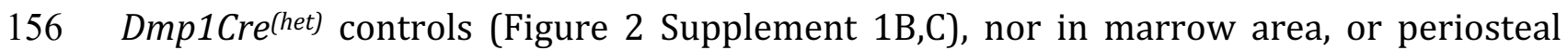

157 circumference (Figure 2 Supplement 1E,I). At 16 weeks, male mice did exhibit a small and

158 significant transient elevation in cortical area and endocortical perimeter; this was no

159 longer detected at 26 weeks (Figure 2 Supplement $1 \mathrm{~F}, \mathrm{H}$ ).

160 Since greater bone width is associated with greater bone strength, we carried out three-

161 point bending tests. Femora from 26 week old male Dmp1Cre.Pthlhf/f(hom) mice could

162 withstand higher loads than age-matched Dmp1Cre(hom) controls, reaching a higher

163 ultimate force (Figure 3A,B) and failure force (Table 3) before breaking. There was no

164 significant difference in ultimate displacement between Dmp1Cre.Pthlhf/f(hom) and control

165 femora (Figure 3C). When these measurements were corrected for bone size, both 14 and

16626 week old male Dmp1Cre.Pthlhf/f(hom) femora showed lower ultimate stress and yield

167 stress, compared to controls (Figure 3D-F). 14 week old male Dmp1Cre.Pthlhf/f(hom)

168 femora had higher ultimate and failure strain than controls (Table 3). 26 week old male

169 Dmp1Cre.Pthlhf/f(hom) femora had lower failure stress (Figure 3G), and reduced toughness

170 and elastic modulus (Table 3) compared to sex- and age-matched controls. This indicates

171 that the greater width of the bones increased bone strength by lowering the stress

172 experienced by the material under three point bending conditions.

173 While ultimate force, failure force, ultimate stress, yield stress, failure stress and 174 toughness were modified in the male mice, these parameters were not changed in females

175 (Figure 3 and Table 3), consistent with their loss of the greater bone width with ageing. 176 Surisingly, femora from female Dmp1Cre.Pthlhf/f(hom) mice achieved a greater yield force 177 and displacement compared to age- and sex-matched controls (Table 3), suggesting a 178 higher elastic deformation. When corrected for bone size, 26 week old female 179 Dmp1Cre.Pthlhf/f(hom) femora had higher yield strain and lower elastic modulus (Table 3), 180 suggesting a more flexible material than controls.

181 Femora from 26 week old heterozygous-bred Dmp1Cre.Pthlhf/f(het) mice did not show any 182 significant difference in mechanical properties compared to Dmp1Cre ${ }^{\text {(het) }}$ controls (Figure 1833 Supplement 1). 


\section{Figure 3}

A
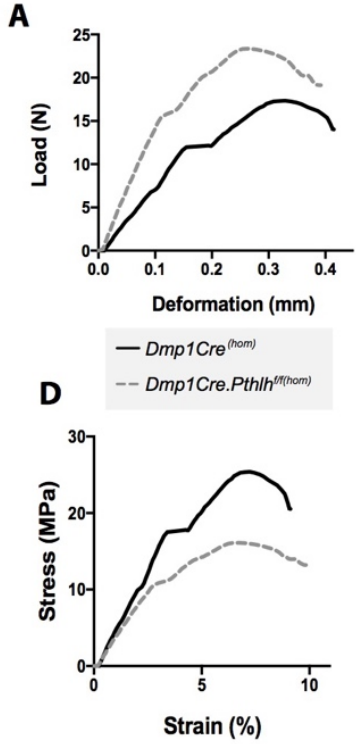

B

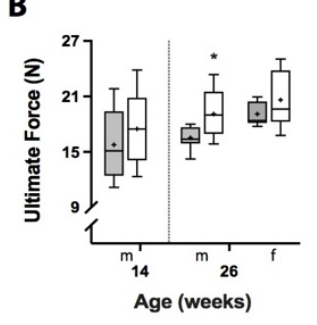

$\mathbf{E}$

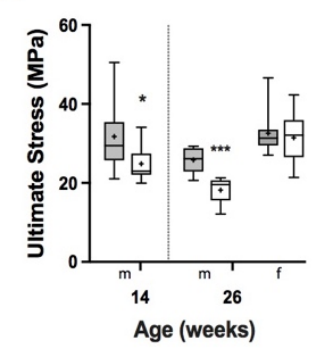

C

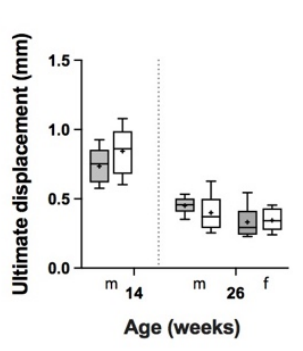

$\mathbf{F}$

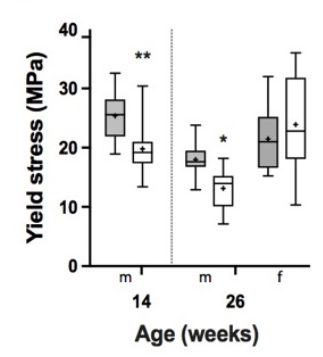

$\square$ Dmp1Cre

口 Dmp1Cre.Pthlh/h(nom)
G

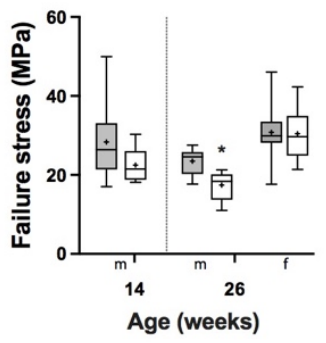

Figure 3: Greater ultimate strength in 26-week old and less stress in 14 and 26-week old male Dmp1Cre.Pthlhf/f(hom) femora measured by three-point bending tests. A: Representative load-deformation curves of 26-week old male Dmp1Cre.Pthlhf/f(hom) and Dmp1Cre(hom) bones. Greater ultimate force (B) and normal ultimate deformation (C) in femora from 26-week old males; no change in 14 week old males and 26 week old females. D: Representative stress-strain curves of 26-week old male Dmp1Cre.Pthlhf/f(hom) and Dmp1Cre (hom) bones, after correction in each sample based on anteroposterior and mediolateral dimensions (shown in Figure 2). Also shown are ultimate stress (E), yield stress (F), and failure stress (G). Data shown as mean (dot), interquartile range (box), median (line) and range, $n=9-10 /$ group. ${ }^{*} \mathrm{p}<0.05,{ }^{* *} \mathrm{p}<0.01$ and ${ }^{* * *} \mathrm{p}<0.001$ compared to age- and sex-matched controls by two-way ANOVA (16 and 26 weeks old) and Student's t-test (14 weeks old).

186 The wide bone phenotype is present at 12 days of age in male and female

187 Dmp1Cre.Pthlhf/f(hom) mice

188 Since we previously observed recombination of PTHrP in the mammary gland in 189 Dmp1Cre.Pthlhf/f(het) mice (7), and deletion of maternal mammary PTHrP is reported to 190 lead to greater bone mass in progeny at 12 days of age (10), we sought to determine 191 whether bone mass was modified in 12 day old Dmp1Cre.Pthlhf/f(hom) mice, and whether 192 maternal milk PTHrP content was reduced. 12 day old mice were assessed from both 193 heterozygous (Dmp1Cre.Pthlhf/f(het) (Figure 4A) and homozygous (Dmp1Cre.Pthlhf/f(hom)

194 (Figure 1A) breeding strategies. 


\section{Figure 4}

A

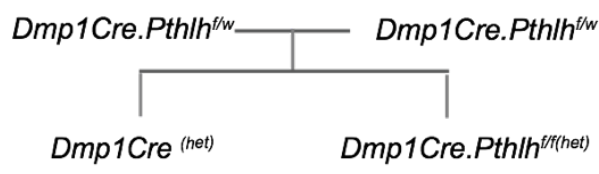

B

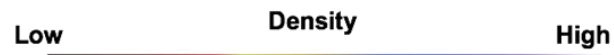

C

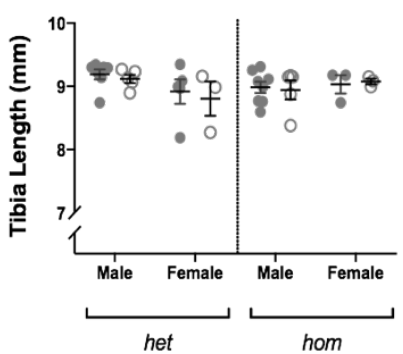

D

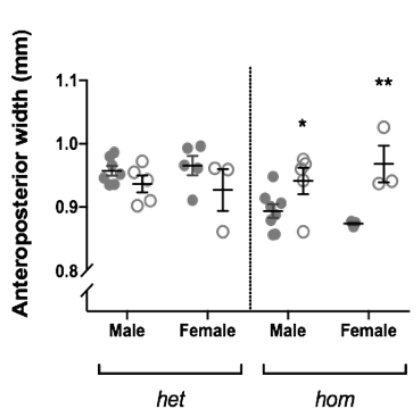

G

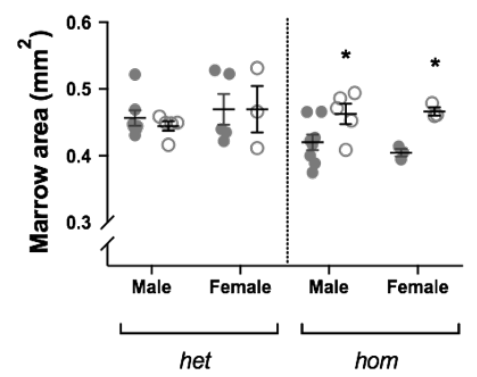

- Dmp1Cre

Dmp1Cre.Pthlh ${ }^{\text {th }}$

Female

$\mathbf{E}$

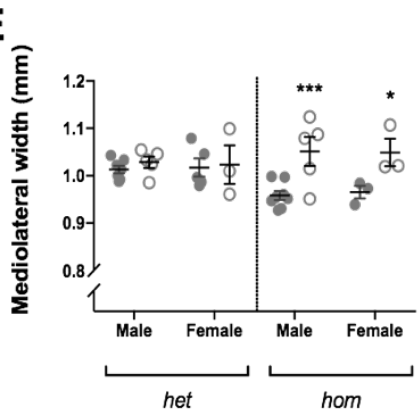

$\mathbf{F}$

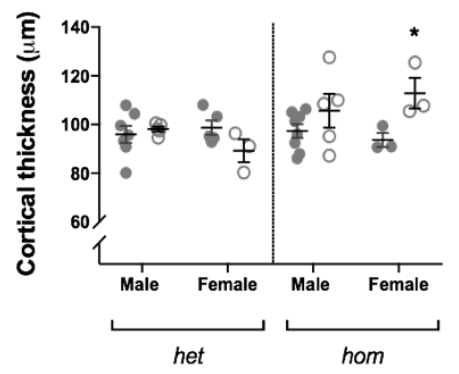

H

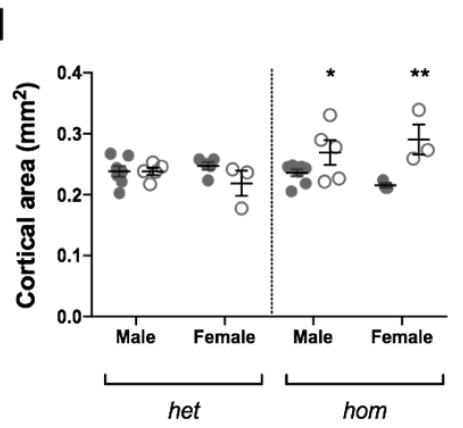

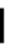

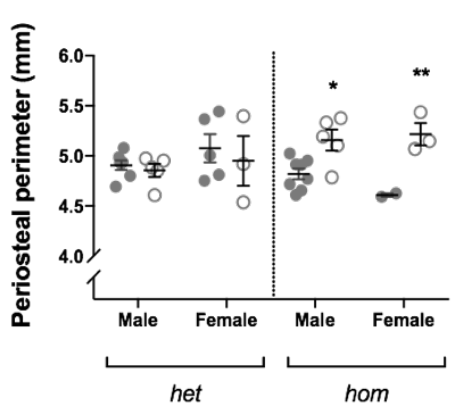

Figure 4: Wide bone phenotype of 12 day old male and female Dmp1Cre.Pthlhf/f(hom) mice. A: Schematic diagram showing heterozygous breeding strategy used for this experiment. B: Representative micro-CT images of cortical bone of 12 day-old Dmp1Cre.Pthlhf/f(hom) tibiae. C-I: Tibial cortical structure of 12 day old Dmp1Cre.Pthlhf/f mice from heterozygous (het) and homozygous (hom) breeders compared to their respective Dmp1Cre controls. Shown are tibial length (C), anteroposterior (D) and mediolateral (E) width, cortical thickness (F), marrow area (G), cortical area $(\mathbf{H})$ and periosteal perimeter (I). Data is shown as mean \pm SEM with individual data points, ${ }^{*} \mathrm{p}<0.01,{ }^{* *} \mathrm{p}<0.01,{ }^{* * *} \mathrm{p}<0.001$ compared to age- and sexmatched controls by two-way ANOVA. 
While no significant differences in cortical dimensions were detected in tibiae from male or female Dmp1Cre.Pthlhf/f(het) mice (from heterozygous breeders), Dmp1Cre.Pthlhf/f(hom) mice (from homozygous breeders) exhibited greater tibial width at 12 days of age (Figure 4B-I) with no difference in tibial length (Figure 4C). Both male and female Dmp1Cre.Pthlhf/f(hom) mice had significantly greater tibial width in the anteroposterior and mediolateral direction, compared to sex-matched Dmp1Cre(hom) (Figure 4B,D,E). Male and female Dmp1Cre.Pthlhf/f(hom) mice also showed greater tibial marrow area (Figure $4 \mathrm{G}$ ), cortical area (Figure 4H), and periosteal perimeter (Figure 4I) compared to sexmatched cousin-bred Dmp1Cre (hom) controls. Female Dmp1Cre.Pthlhf/f(hom) also had greater cortical thickness than female Dmp1Cre(hom) controls (Figure 2F). No significant difference was observed in trabecular structure between Dmp1Cre.Pthlhf/f(hom) mice and their Dmp1Cre(hom) sex-matched controls (Table 4), suggesting that this aspect of the phenotype was secondary to the increase in bone width.

211 Although PTHrP gene recombination was detected in mammary tissue from

212 Dmp1Cre.Pthlhf/f(het) mice (7), milk PTHrP levels, measured either by radioimmunoassay 213 or bioassay, and milk protein levels were not significantly altered in Dmp1Cre.Pthlhf/f(het) 214 mice compared to controls (Table 5).

\section{The Dmp1Cre.Pthlhf/f(hom) wide-bone phenotype exists in utero}

216 Since no change in milk PTHrP could explain the phenotype at 12 days of age, we 217 determined whether the phenotype existed in utero by assessing embryonic bone size.

218 Consistent with our observations at 12 days, embryonic Dmp1Cre.Pthlhf/f(hom) femora 219 (E18.5) were wider in both anteroposterior and mediolateral dimensions, and exhibited 220 a higher moment of inertia compared to Dmp1Cre ${ }^{(\text {hom })}$ controls (Figure 5A, D-I). Greater bone area and tissue area of Dmp1Cre.Pthlhf/f(hom) embryos confirmed that their femora are wider than controls (Figure 5 Supplement 1). This greater bone width was observed along the full length of the bone, including both metaphysis and the diaphysis.

The length of the mineralized portion of Dmp1Cre.Pthlhf/f(hom) femora, measured by micro-CT, was also greater than Dmp1Cre(hom) controls (Figure 5B). Micro-computed tomography cannot detect the full length of the bone at this age, as the cartilage ends are not yet mineralized prior to birth. Total femoral length measured in Alizarin Red/Alcian Blue stained samples showed no difference between Dmp1Cre.Pthlhf/f(hom) and Dmp1Cre (hom) controls (Figure 5C), indicating that Dmp1Cre.Pthlhf/f(hom) embryos have normal bone length, but accelerated mineralization in utero.

231 Heterozygous-bred Dmp1Cre.Pthlhf/f(het) embryos did not show any significant difference

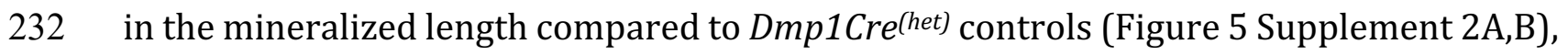
233 nor in the bone width or structure (Figure 5 Supplement 2C-K). 


\section{Figure 5}

A

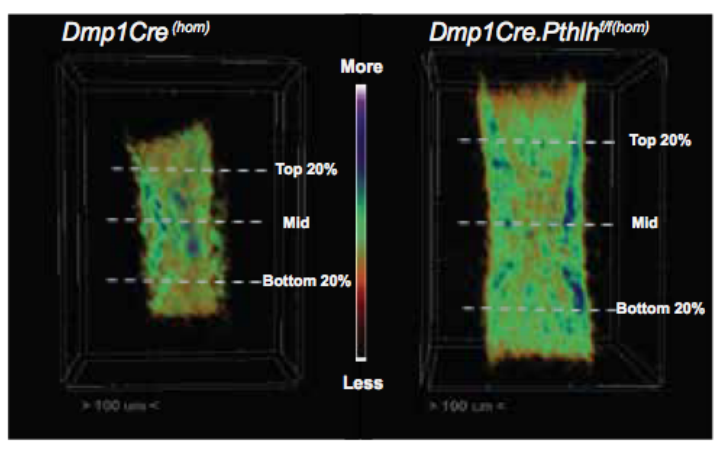

- Dmp1Cre (hom) $_{\text {D }} \quad$ Dmp1Cre.Pthlh/f(hom)

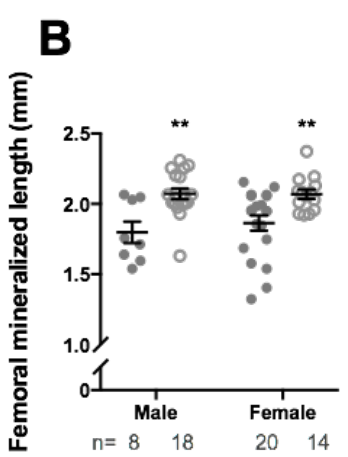

C

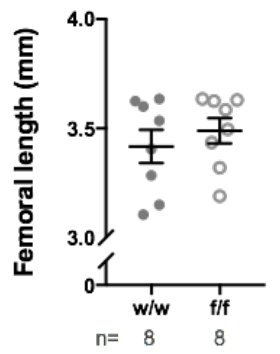

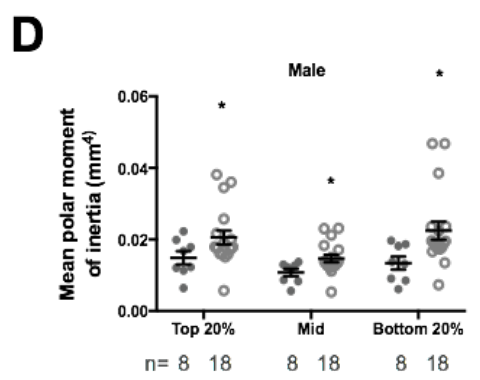

E

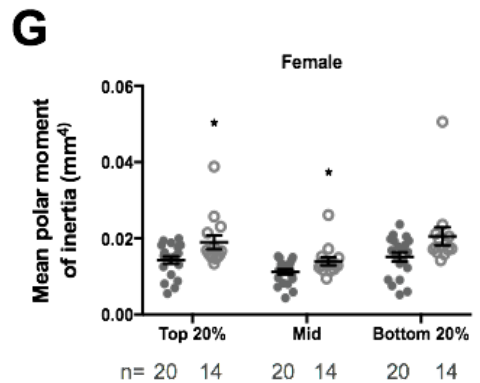

H
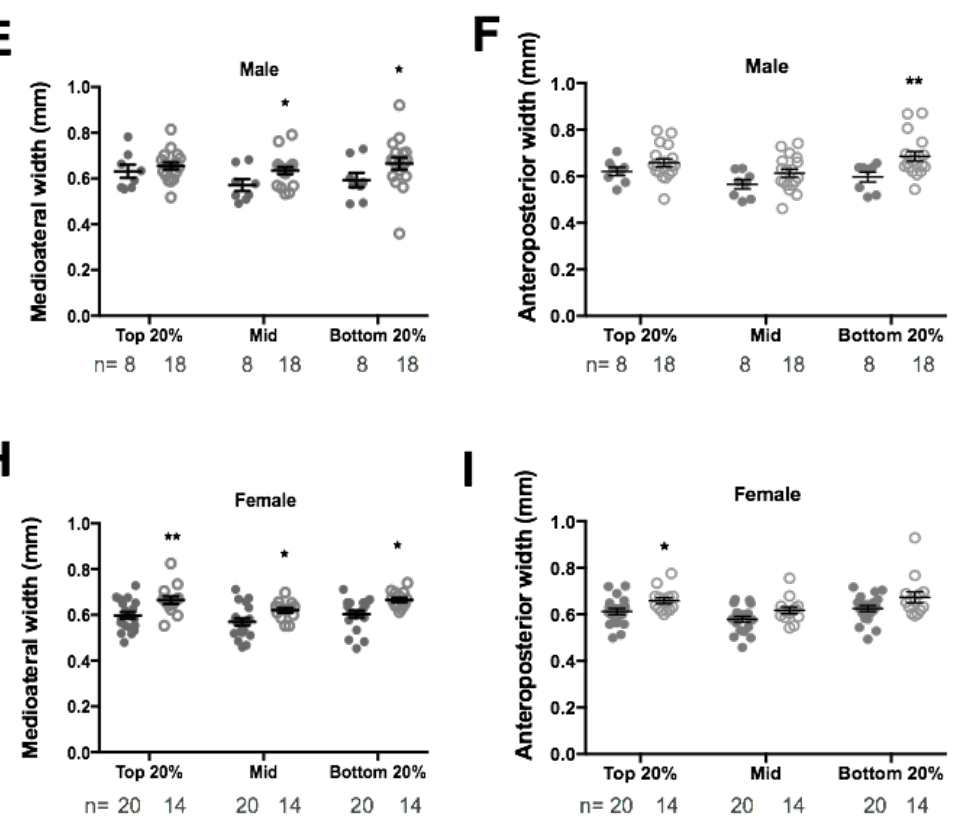

Figure 5: Accelerated bone development of E18.5 Dmp1Cre.Pthlhf/f(hom) femora.

A) representative images of Dmp1Cre.Pthlhf/f(hom) and Dmp1Cre(hom) femora at E18.5, showing bone mineral density. B) Femoral mineralized length, C) total femoral length, D-I: Mean polar moment of inertia (D, G), mediolateral (E, H), and anteroposterior widths (F, I) of Dmp1Cre.Pthlhf/f(hom) and Dmp1Cre (hom) at three different locations shown in panel A: at $20 \%$ of the mineralized length distal to the proximal end of the mineralized region (Top 20\%), at the midshaft (Mid), and at $20 \%$ of the mineralized length proximal to the distal end of the mineralized region (Bottom 20\%). Data is shown as mean \pm SEM with individual data points, ${ }^{*} \mathrm{p}<0.05$, ${ }^{* *} \mathrm{p}<0.01$, and ${ }^{* * *} \mathrm{p}<0.001$ compared to controls by two-way ANOVA (B-H). 
238 Since we observed increased bone width in utero in Dmp1Cre.Pthlhf/f(hom) mice, and PTHrP

239 is produced by uterus and decidua $(11,12)$, we sought to determine whether PTHrP

240 expression is modified in placenta or decidua from Dmp1Cre.Pthlhf/f(hom) mice. Consistent

241 with a lack of change in overall bone growth, there were no significant differences in body

242 weight, placental weight, or body to placental weight ratios between Dmp1Cre.Pthlhf/f(hom)

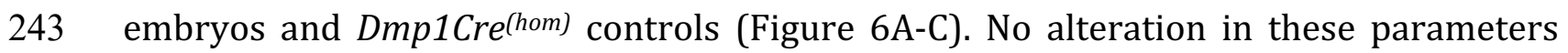

244 were observed in Dmp1Cre.Pthlhf/f(het) embryos and placenta compared to littermate

245 Dmp1Cre.Pthlhw/w(het) (Figure 6 Supplement 1A-D). This suggests the increased bone

246 width of Dmp1Cre.Pthlhf/f(hom) embryos is not caused by changes in placental efficiency.

247 PTHrP staining of decidua and placenta showed positive staining for PTHrP in both the 248 decidua and the spongiotrophoblast layer (junctional zone) of the placenta (Figure 6D). 249 No PTHrP was detected in the placental labyrinth zone. PTHrP staining in decidua from 250 mothers of Dmp1Cre.Pthlhf/f(hom) mice was not as strong as that observed in decidua from 251 mothers of Dmp1Cre $e^{(\mathrm{hom})}$ mice (Figure 6D,E). Quantification revealed a significant 252 reduction in PTHrP staining at all intensities in decidua from mothers of 253 Dmp1Cre.Pthlhf/f(hom) mice, but no change in PTHrP staining frequency in the 254 spongiotrophoblast zone of the adjacent placenta (Figure 6F). IgG isotype control had 255 minimal intensity in both regions. No alteration in PTHrP staining frequency was 256 observed in in decidua adjacent to Dmp1Cre.Pthlhf/f(het) placenta compared to littermate 257 Dmp1Cre.Pthlhw/w(het) (Figure 6 Supplement 1E). This suggests off-target effects of 258 Dmp1Cre have led to reduced PTHrP protein production by decidual cells.

259 We also examined the morphology of decidua from samples adjacent to 260 Dmp1Cre.Pthlhf/f(hom) placenta. The decidual cells from mothers of Dmp1Cre.Pthlhf/f(hom) 261 embryos appeared more compact than in Dmp1Cre(hom) decidua, suggesting impaired 262 decidual cell maturation (Figure 6G,H). Total decidual area was significantly less in 263 samples from mothers of Dmp1Cre.Pthlhf/f(hom) embryos than Dmp1Cre(hom), but the area 264 of the spongiotrophoblast zone was not significantly modified (Figure 6I). No change in 265 decidual size was detected in decidua adjacent to Dmp1Cre.Pthlhf/f(het) placenta compared 266 to littermate Dmp1Cre.Pthlhw/w(het) controls (Figure 6 Supplement 1F,G). This suggests 267 PTHrP may act locally within the decidua to promote decidual cell maturation, and this may cause increased bone width growth of Dmp1Cre.Pthlhf/f(hom) mice in utero. 
Figure 6
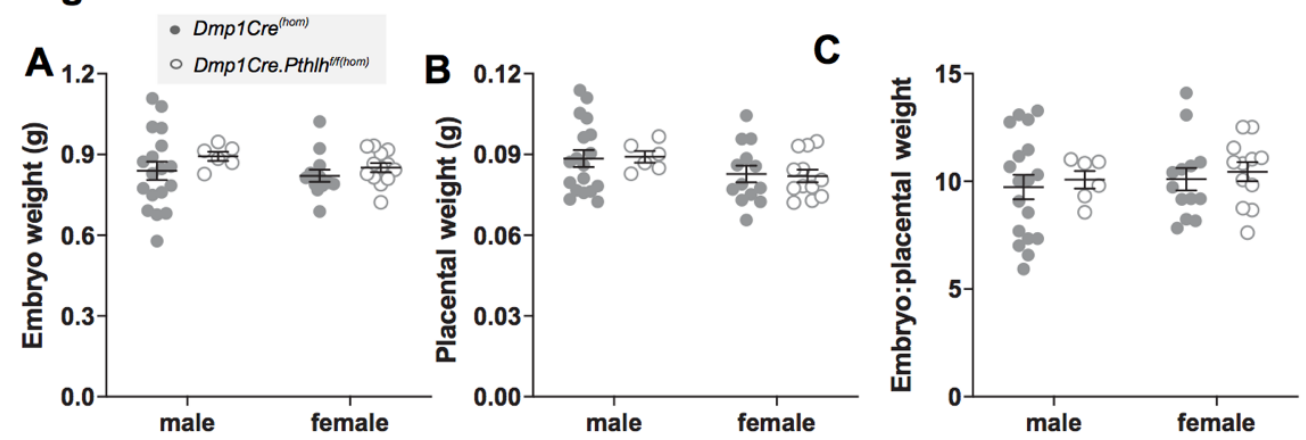

D

$\mathbf{F}$

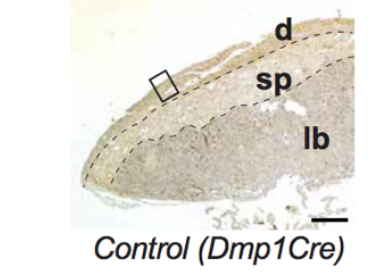

$\mathbf{E}$
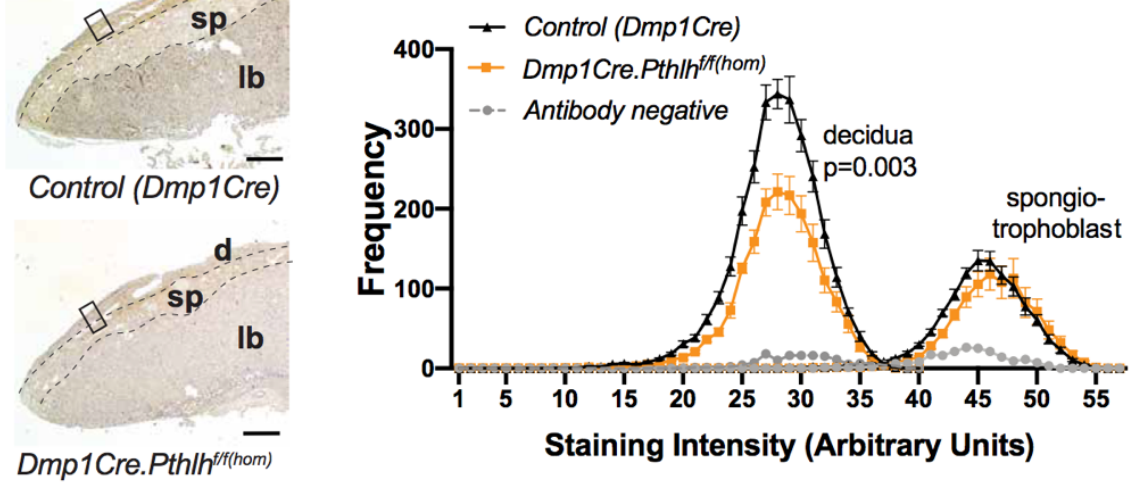

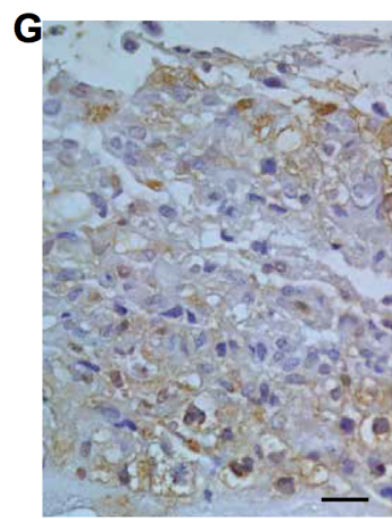

Control

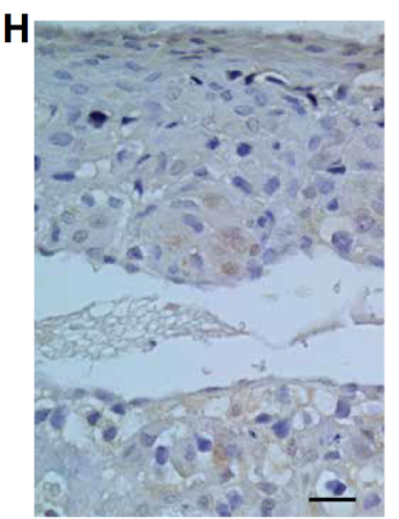

Dmp1Cre.Pthlh $h^{f f(h o m)}$
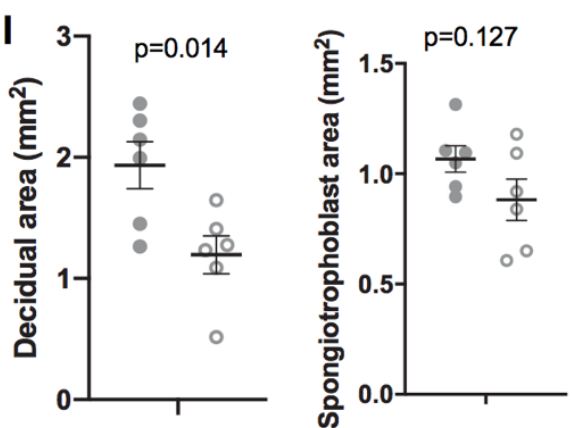

- Control (Dmp1Cre)

- Dmp1Cre.Pthl/ht(hom)

Figure 6: Decreased decidual PTHrP and impaired decidualization of Dmp1Cre.Pthlhf/f

mice at E17.5. Embryo weight (A), placental weight (B) and embryo to placental weight ratio (C) of Dmp1Cre.Pthlhf/f(hom) and Dmp1Cre(hom). (D-H) Immunostaining for PTHrP in samples of placenta and decidua from Dmp1Cre.Pthlhfff(hom) and Dmp1Cre(hom) embryos at E17.5; IgG control staining was measured in images of both zones. Decidua (d), spongiotrophoblast (sp) and labyrinth (lb) zones are shown in low power images (D,E). Scale bar $=1 \mathrm{~mm}$. Frequency of PTHrP stained objects segregated by staining intensity in the spongiotrophoblast layer and decidua from Dmp1Cre.Pthlhfff(hom) and Dmp1Cre (hom) embryos. (F,G) High power images of decidua. Scale bar $=20$ micron. (I) Quantitation of total decidual area and spongiotrophoblast area; mean \pm SEM with individual data points, ${ }^{*} \mathrm{p}<0.05$, ${ }^{* *} \mathrm{p}<0.01$ compared to controls by one-way ANOVA. 


\section{Discussion}

This study identifies that off-target effects of Dmp1Cre-mediated recombination led to reduced decidual PTHrP. Reduced PTHrP level in the decidua is associated with increased embryonic bone radial growth and mineralization in utero. This wide bone phenotype is observed in both male and females at 12 days of age, and is sustained until at least 6 months of age in male, but not female, skeletons (Figure 7). These effects of reduced decidual PTHrP on bone size, trabecular bone mass, and bone strength dominates over the previously reported effects of reducing endogenous PTHrP in osteocytes, which suppressed bone formation and reduced trabecular bone mass of young adult mice (7). This suggests that locally produced PTHrP is essential for normal decidualization, and through these actions influences embryonic bone growth. This indicates an additional role for PTHrP in maternal physiology.

\section{Figure 7}

\section{Heterozygous breeders}
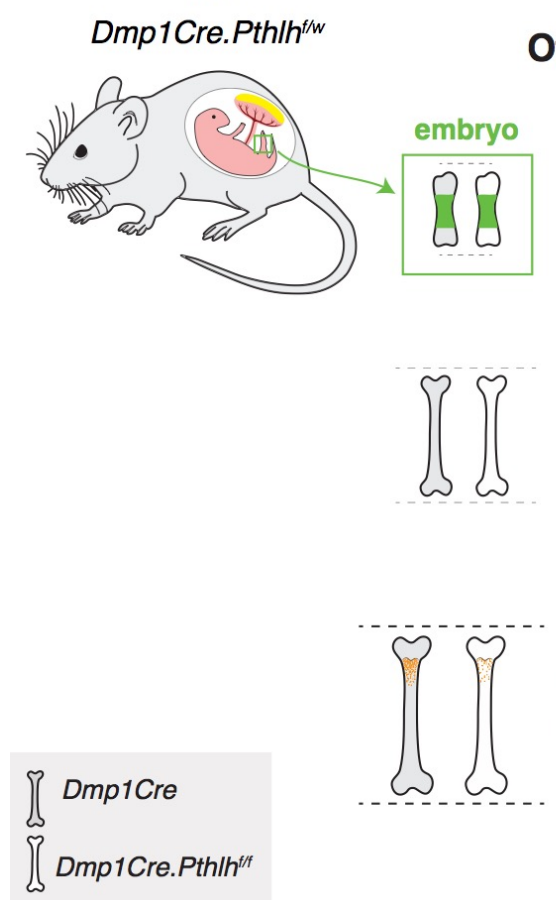

\section{Homozygous breeders}

Offspring age
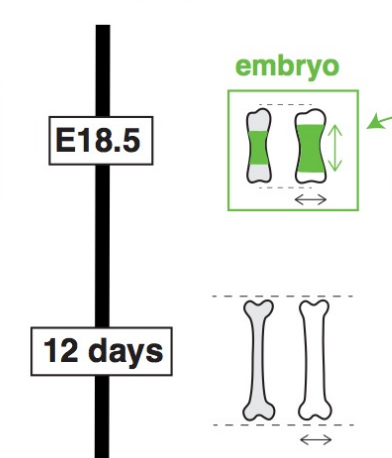
Dmp1Cre.Pth/h ${ }^{\text {t/t }}$

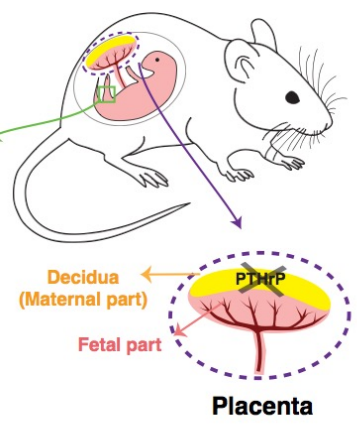

Male
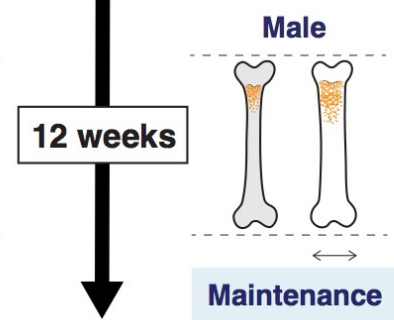

Female

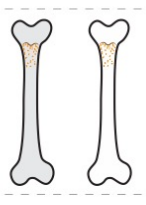

Recovery
283

Figure 7: Decidual PTHrP determines bone width in progeny. Mice from breeders heterozygous for PTHrP (Dmp1Cre.Pthlhf/w breeders) had normal bone size (length and width) compared to their sex- and age-matched controls, but lower adult trabecular bone mass. Decidual PTHrP may limit fetal skeletal development and radial growth, independent of longitudinal growth. Mothers of Dmp1Cre.Pthlhf/f(hom) mice (which are Dmp1Cre.Pthlhf/f(het) ) had lower levels of decidual PTHrP, leading to wider long bones in Dmp1Cre.Pthlhf/f(hom) progeny. This phenotype was observed not only in embryos and neonatal mice, but also in adult male mice. Adult male mice also showed high trabecular bone mass compared to their sex-matched Dmp1Cre controls. 
Our data contrasts the role of maternal-derived PTHrP with endogenous osteocytic PTHrP. Dmp1Cre.Pthlhf/f(hom) mice exhibited wider long bones during late embryogenesis, at 12 days of age in both males and females, and during adulthood in males, leading to greater bone strength at 26 weeks of age. This phenotype was not observed in Dmp1Cre.Pthlhf/f(het) mice at any time point studied. In addition to their increased bone width, adult male Dmp1Cre.Pthlhf/f(hom) mice had high trabecular bone mass, in contrast to the osteopenia observed in Dmp1Cre.Pthlhf/f(het) mice at 12 weeks of age (7). The requirement for decidual PTHrP therefore dominates the requirement for endogenous osteocytic PTHrP. The influence of decidual PTHrP in restricting bone width also contrasts with the role of endogenous PTHrP from the embryo-derived portion of the placenta, which promotes placental calcium transport and skeletal development, as indicated by reduced bone length in Pthlh null embryos (5).

The role of decidual PTHrP has not previously been investigated. PTHrP is produced within the decidua, myometrium, amnion, and chorion, where it dilates the uterine and placental vasculature and inhibits myometrial contractions (13-15). Here we showed PTHrP is expressed by decidua and spongiotrophoblast layer (or junctional zone), whereas labyrinth zone of placenta had undetectable levels of PTHrP. Decidua from mothers of Dmp1Cre.Pthlhf/f(hom) mice had significantly lower PTHrP staining, but there was no change in placental PTHrP staining, supporting a maternal origin of the defect. Decidua from mothers of Dmp1Cre.Pthlhf/f(hom) mice were also smaller in size and decidual cell morphology was modified. The smaller decidua and reduced size of decidual cells in mothers of Dmp1Cre.Pthlhfff(hom) mice was a surprising finding in this study. This may reflect reduced decidual cell differentiation, since decidual cells are enlarged during decidualization $(16,17)$. Alternatively, it may reflect decidual cell atrophy in mothers of Dmp1Cre.Pthlhf/f(hom) mice. Since previous studies reported that PTHrP repressed decidualization of human uterine fibroblast cells (18), that intrauterine injection of PTH/PTHrP receptor antagonist from day 6 to 13 post coitum (after induction of decidua) increased decidualisation in rats (19), and Pthlh mRNA levels are downregulated during decidualization in primary endometrial stromal cells (20), we suggest the reduced size of decidual cells at day E17.5 may reflect early commencement of atrophy. How this drives the increased radial growth and mineralization in the Dmp1Cre.Pthlhf/f(hom) embryo remains to be established.

It is very surprising that Dmp1-Cre targeted recombination had an influence on decidua. Although the Dmp1-Cre mouse is widely used as an osteocyte and late-osteoblast conditional knockout mouse, multiple off-target tissues have been reported. These include our previous report of recombination in the mammary gland (7). We and others have shown recombination in skeletal muscle and certain brain cells (7), and reporter genes have also shown Dmp1-Cre expression in preosteoblasts, a subset of bone marrow stromal cells, and gastrointestinal mesenchymal stromal cells (21). To date, there is no report that Dmp1-Cre targets decidua, which is a transient uterine tissue. We previously tested non-pregnant uterus, and found that Dmp1-Cre recombination did not occur (7). 
The expression of Dmp1-Cre in decidua has major implications for the design and

328 reporting of experiments utilizing Dmp1Cre for gene deletion. However, this clearly 329 depends on the function of the targeted gene. For example, although gp130, and its 330 inhibitor protein SOCS3, are expressed in murine decidua $(22,23)$, homozygous-bred 331 Dmp1Cre.gp130f/f mice (8) and Dmp1Cre.Socs3f/f mice (9) showed phenotypes similar to 332 that of heterozygous-bred mice of the same genotype $(9,24)$.

333 Although decidua and placenta provide nutrition to promote embryonic and placental 334 weight gain in utero (25), and these effects influence adult health, including bone mass 335 (26), there was no change in total embryo or placental weight in Dmp1Cre.Pthlhf/f(hom) 336 embryos. This suggests maternal contributors to general embryonic nutrition, such as uteroplacental blood flow and nutrient transport across the placenta controlled by (for example) growth hormone, IGF-I, insulin, and glucocorticoids, are unlikely to contribute to the phenotype we observe. The influence of decidual PTHrP on growth appears to be specific to the skeleton. The alteration in the morphological features of decidua might be associated with changes in its function, resulting in changes in skeletal development. Whether decidual PTHrP, like embryonic placental PTHrP regulates placental calcium transport or fetal PTH levels $(27,28)$ or other local regulators of bone development, which are known to regulate fetal bone development (29), or it acts systematically to regulate bone radial growth remains unknown.

The increased mineralization length and greater bone width of Dmp1Cre.Pthlhf/f(hom) embryos and adult male Dmp1Cre.Pthlhf/f(hom) mice along the full length of the bone suggests that the maternal influence on bone widening determines radial expansion of the embryonic cartilage anlagen, the early cartilage model of the developing bone, rather than inducing periosteal apposition at the diaphysis. Maternal PTHrP may therefore limit radial expansion of chondrocytes during cartilage anlage development, and may suppress signalling pathways that promote expansion of chondrocytes at growth plate. This effect contrasts with actions of embryo-derived PTHrP from the placenta (5) and cartilage (30, 31) to stimulate longitudinal bone growth. Although longitudinal bone growth has been studied widely, very little is known about signaling pathways orchestrating radial growth. There are two non-mutually-exclusive theories describing how bone radial width is determined: the "mechanostat" theory suggests that bone size and shape are adapt to mechanical strain $(32,33)$, while the "sizostat" theory suggests a set of genes regulates bone width to reach a pre-programmed setting (34). Although different genomic markers have been correlated with bone size and bone shape (35), no specific genes or molecular pathways have yet been described as major determinants of cortical bone diameter. Our data suggests that decidual PTHrP is a determinant for the cortical width sizostat.

Although both male and female Dmp1Cre.Pthlhf/f(hom) mice had wider long bones at 12 days of age, this phenotype was retained through to adulthood only in males, suggesting that the mechanisms controlling continued radial growth and bone width are sexdependent. Although placental nutrition has sexually dimorphic effects on embryo 
growth (36), we did not observe sex differences in this study until after 12 days, again emphasizing that the wide bone phenotype is unlikely to relate to placental nutrition. Post-pubertal sex differences in cortical diameter are common to all mammals (36-45), with females having narrower bones than males, however the molecular mechanisms driving this sexual dimorphism remain largely unknown; this mouse model may therefore shed new light on the mechanisms that contribute to this sexual dimorphism. The retention of this phenotype in males, but not females, suggests that hormonal changes at puberty in females may slow their radial growth. While most studies investigating sexual dimorphism in murine bone width have focused on periosteal growth at the diaphysis, our results suggest that differences between males and females in cortical width might also arise from radial expansion of the growth plate. Estradiol is known to slow longitudinal growth: a previous study has shown that ovariectomy increased tibial length and increased chondrocyte proliferation (46), and 17betaestradiol treatment of 26-day-old female and male rats led to shorter tibial length and an early reduction in growth plate longitudinal width (46). Testosterone also affects chondrocytes: local injection of testosterone into the tibial epiphyseal growth plate of castrated growing male rats significantly increased epiphyseal growth plate length (47). Furthermore, while the perinatal testosterone surge is required for adult bone length, bone width is determined by post-pubertal testosterone (48). The cellular and intracellular pathways by which estradiol and/or testosterone differentially affect growth plate radial growth in control and Dmp1Cre.Pthlhf/f(hom) mice remains to be investigated.

In Dmp1Cre.Pthlhf/f(hom) mice, greater cortical width was not associated with greater total bone length. To our knowledge, this is the first evidence of changes in bone diameter independent of cortical thickness, longitudinal growth, and total body weight gain. All previously reported mouse models with changes in bone diameter also showed widespread skeletal development defects such as reduced bone length and width or altered cortical thickness (48-51). For example, mice lacking the endogenous nuclear localization sequence and C-terminus of PTHrP displayed retarded growth with lower body weight and total skeletal size at the age of 2 weeks (50), while Insulin-like growth factor I null $(\operatorname{Igf1} \%$ mice displayed smaller body size, shortened femoral length and reduced cortical thickness compared to wild type littermates $(51,52)$. Having altered radial, but not longitudinal, growth makes the Dmp1Cre.Pthlhf/f(hom) mouse an appealing model for studying specific mechanisms underlying radial bone growth. Discovering such mechanisms might open new therapeutic avenues for improving cortical bone strength and reducing fracture risk in growing children and in adults. If there is no change in material content, bones with wider cortices are more resistant to fracture $(34,53)$. Indeed, adult male Dmp1Cre.Pthlhf/f(hom) bones could withstand greater force, and experienced lower ultimate stress, before failure in three point bending tests compared to age- and sex-matched controls.

Although we detected PTHrP recombination in the mammary glands (7), milk PTHrP 
408

409

410

411

412

413

414

415

416

417

418

419

420

421

422

423

424

425

426

427

428

429

430

431

432

433

434

435

436

437

438

439

440

441

442

443

444

445

446

447 levels were not significantly modified, and the wide-bone phenotype predated the commencement of suckling, indicating that a change in mammary supply of PTHrP is unlikely to cause the wide-bone phenotype we observed in Dmp1Cre.Pthlhf/f(hom) mice. We had thought that this may have been a possibility since we previously noted $\mathrm{Dmp1Cre-}$ driven PTHrP recombination in the mammary gland (7), and suckling pups from mice lacking PTHrP in the milk supply (BLG-Cre/PTHrPlox/-) had higher ash calcium content, indicating greater bone mass, compared to controls at day 12 of lactation (10). The normal levels of PTHrP in milk in mothers of Dmp1Cre.Pthlhf/f(hom) mice suggests that the mammary cells expressing Dmp1Cre are not the mammary epithelial cells that secrete PTHrP to the milk (54), and are different to those targeted in the BLG-Cre/PTHrPlox/model $(10,55,56)$. Another possibility is that the level of PTHrP recombination was too low in mammary tissues to modify milk PTHrP production.

In conclusion, decidual PTHrP limits trabecular bone mass, bone geometry and strength, not only of neonatal mice, but also of adult male mice. Dmp1Cre.Pthlhf/f(hom) embryos had accelerated skeletal development, with more mineralized and wider femora at E18.5. Although this effect was observed in both males and females in neonates, it was retained through to adulthood only in male mice. This indicates that maternal PTHrP limits bone growth, and this has a life-long influence on bone mass, shape and strength in male progeny.

\section{Materials and Methods}

\section{Animal experiments}

Dmp1Cre.Pthlhf/f(het) mice have been described previously (7) and were bred from Dmp1Cre (Tg(Dmp1-cre) $\left.)^{1 \mathrm{Jqfe}}\right)$ mice (containing the Dmp1 10-kb promoter region) provided by Lynda Bonewald (University of Kansas, Kansas City, USA) (57), and Pthlhflox (Pthl $h^{\mathrm{tm} 1 \mathrm{Ack}}$ ) mice by Andrew Karaplis (McGill University, Montreal) (58) with LoxP sites spanning Pthlh exon III (7).

Two breeding strategies were used in this study (Figure 1A). Initially, mice hemizygous for Dmp1Cre were crossed with Pthlhf/f mice to generate Dmp1Cre.Pthlhf/w breeders. These were used to generate hemizygous-bred PTHrP deficient (Dmp1Cre.Pthlhf/f(het)) and Dmp1Cre mice, as in our previous study (7). Dmp1Cre.Pthlhf/f(hom) mice were generated from breeding pairs that were both Dmp1Cre.Pthlhf/f(het). Cousin-matched Dmp1Cre mice were bred to generate homozygous-bred Dmp1Cre cousin controls (denoted Dmp1Cre(hom)). Adult mice were collected at 14 weeks (male only), and at 26 weeks (both male and female) after an in vivo microCT scan at 16 weeks of age. Sample sizes used were based on our previous studies; no explicit power analysis was used.

12 day old pups were collected from both homozygous and heterozygous breeders. For the milk collection, mice were mated at 6 weeks of age, and after the mice became pregnant for the first time, males were removed. At day 12 of the (first) lactation, dams 
were anesthetized and injected with 1 IU of oxytocin (Sigma) (59). After 5 minutes, milk was collected and then kept at $-80^{\circ} \mathrm{C}$.

Dmp1Cre.Pthlhf/f(hom) and Dmp1Cre(hom) embryos and matching placenta/decidua were collected (4-7 litters/genotype) at embryonic day (E)18.5 of first pregnancy, and Dmp1Cre.Pthlhf/f(het) embryos, placenta and decidua were collected at E17.5 of first pregnancy. The sex of embryos was determined by PCR, as described previously (60). All mice were housed at the St Vincent's BioResources Centre, in a $12 \mathrm{~h}$ light and dark cycle and provided food and water ad libitum. St. Vincent's Health Animal Ethics Committee approved all animal procedures. Terminal blood samples were collected by cardiac puncture exsanguination and sera kept at $-80^{\circ} \mathrm{C}$.

\section{Micro-computed tomography (micro-CT)}

Micro-CT was carried out on samples from E17.5, E18.5, 12 days, 14 and 26 weeks of age. The observer was blinded to genotype and sex of all samples at the time of analysis. 26 week old mice were also anaesthetized and scanned by in vivo micro-CT at 16 weeks of age. After collection, embryos were fixed in 95\% ethanol for 5 days. Femora of 14 and 26 week old mice, and tibiae of 12 day old mice were fixed overnight in $4 \%$ paraformaldehyde at $4{ }^{\circ} \mathrm{C}$, then stored in $70 \%$ ethanol until further analysis. Femoral and tibial morphology and microarchitecture were assessed using the Skyscan 1076 (E18.5, 12 days, 14 and 26 weeks of age) or 1276 (E17.5) micro-CT system (Bruker, Aartselaar, Belgium), as described previously (61) with the following modifications.

For micro-CT analysis at E17.5 and E18.5, embryos were scanned at $55 \mathrm{kV}$ and $200 \mathrm{~mA}$, and $48 \mathrm{kV}$ and $208 \mathrm{~mA}$, respectively. Projections were acquired over a pixel size of $5 \mu \mathrm{m}$ and $9 \mu \mathrm{m}$, respectively. Image slices were then reconstructed by NRecon (Bruker, version 1.7.1.0) with beam-hardening correction of $35 \%$, ring artifact correction of 6 , smoothing of 1 , and defect pixel masking of $50 \%$. The length of mineralized bone was measured in each femur. Femoral cortical structure was analyzed at three sites, based on the extent of mineralised femur: i) $20 \%$ of the mineralized length distal to the proximal end of the mineralized region (metaphysis; Top 20\%); ii) Midshaft (Mid); iii) $20 \%$ of the mineralized length proximal to the distal end of the mineralized region (Bottom 20\%). Automatic adaptive thresholding was used for each sample.

Tibiae from 12 day old mice were scanned at $37 \mathrm{kV}$ and $228 \mathrm{~mA}$. Regions of interest (ROI) commenced at a distance equal to $30 \%$ of the tibial length down the growth plate and an ROI of $10 \%$ of the tibial length was analyzed. The lower adaptive threshold limit used for cortical analysis was equivalent to $0.58 \mathrm{~g} / \mathrm{mm}^{3}$ Calcium hydroxyapatite (CaHA).

Femora from 14, 16 and 26 week old mice were scanned at $45 \mathrm{kV}$ and $220 \mathrm{~mA}$. For trabecular and cortical analyses, ROI commenced at a distance equal to $7.5 \%$ or $30 \%$, respectively, of the total femur length proximal to the distal end of the femur; for each, an ROI of $15 \%$ of the total femur length was analyzed. For 14 week old mice, the lower 
adaptive threshold limits for trabecular and cortical analysis were equivalent to 0.34 $\mathrm{g} / \mathrm{mm}^{3}$ and $0.75 \mathrm{~g} / \mathrm{mm}^{3}$ CaHA, respectively. For 16 week old mice, the lower adaptive threshold for trabecular and cortical analysis were equivalent to $0.30 \mathrm{~g} / \mathrm{mm}^{3}$ and 0.64 $\mathrm{g} / \mathrm{mm}^{3} \mathrm{CaHA}$, respectively. For 26 week old mice, the lower adaptive threshold for trabecular and cortical analysis were equivalent to $0.33 \mathrm{~g} / \mathrm{mm}^{3}$ and $0.76 \mathrm{~g} / \mathrm{mm}^{3} \mathrm{CaHA}$, respectively. For trabecular analysis in the $5^{\text {th }}$ lumbar vertebrae (L5), an ROI of half the height of the bone (vertically centered) with a diameter $2 / 3$ the width of the vertebral body was analysed.

\section{Histomorphometry}

Tibiae from 14 week old mice were embedded in methylmethacrylate and sectioned at 5 $\mu \mathrm{m}$ thickness for histomorphometric analysis, as previously described (62). The observer was blinded to genotype and sex of all samples during analysis. To determine bone formation rates, calcein was injected intraperitoneally $(20 \mathrm{mg} / \mathrm{kg}$ ) at 7 and 2 days before tissue collection. Sections were stained with Toluidine blue or Xylenol orange, as described (63). Static and dynamic histomorphometry of trabecular bone surfaces was carried out in the secondary spongiosa of the proximal tibia using the OsteoMeasure system (Osteometrics Inc., USA).

\section{Three-point bending test}

Mechanical properties of femora were derived from three-point bending tests using a Bose Biodynamic 5500 Test Instrument (Bose, DE, USA), as described previously (64). The observer was blinded to genotype and sex of all samples during analysis. Once wholebone properties were determined, tissue-level mechanical properties were calculated using micro-CT analysis of the mid-shaft (1).

\section{Biochemical assays}

Cross-linked C-telopeptides of type I collagen (CTX-1) were measured in duplicate with the IDS RatLaps enzyme immunoassay (Abacus, Berkeley, CA, USA) in serum collected from mice fasted overnight. Serum levels of procollagen type $1 \mathrm{~N}$ propeptide (P1NP) were measured in duplicate using IDS Rat/Mouse PINP EIA kit (Abacus, Berkeley, CA, USA).

To measure milk PTHrP content, amino-terminal PTHrP RIA was carried out as previously described, with a sensitivity of $2 \mathrm{pM}$ (65). Milk was diluted 1:500 in assay buffer prior to measurement of PTHrP. Milk PTHrP levels were also bioassayed as the cAMP generated in response to treatment of UMR106-01 cells, using PTH(1-34)-induced cAMP response as a standard curve (66). Replicate cell cultures in 24-well plates were incubated in cell culture medium with $1 \mathrm{mM}$ isobutylmethylxanthine (IBMX) added. After treatment for 12 mins with 1:8 diluted milk samples, cAMP was measured by removing medium and adding acidified ethanol, drying, reconstituting in assay buffer and cAMP assay as described (67). cAMP was then corrected for total protein content of the milk, measured by Pierce BCA protein assay kit (Thermo Fisher Scientific). For this, milk was diluted 1:400 in PBS and absorbance was measured at OD562nm using the Polarstar 
Optima+ and a bovine serum albumin standard curve.

$526 \quad$ Embryo skeletal staining

527 Alcian blue and Alizarin red S staining was carried out on E18.5 embryos, as described 528 previously (68). Embryos were fixed in 95\% ethanol for 5 days after skin removal. 529 Remnant skin and viscera were dissected as much as possible, followed by defatting in 530 acetone for 2 days. Thereafter, they were stained for 4 days at $40{ }^{\circ} \mathrm{C}$ in freshly prepared 531 staining solution: $0.3 \%$ Alcian blue in 70\% ethanol - 1 volume; $0.1 \%$ alizarin red S in $95 \%$ ethanol - 1volume; glacial acetic acid - 1 volume; 70\% ethanol - 17 volumes. After washing in distilled water for 2 hours, they were cleared with $2 \%$ potassium hydroxide (KOH) for

5342 days. Afterwards, they were put in $20 \%$ Glycerol in $1 \% \mathrm{KOH}$ until skeletons were clearly 535 visible, then successively placed into 50\%, 80\% and $100 \%$ glycerol solutions in $1 \% \mathrm{KOH}$ 536 for 2 days each. Femoral length was determined by measuring the distance between 537 femoral head and distal end through a dissecting microscope, and an average of right and 538 left femur lengths in each embryo was calculated.

\section{Immunohistochemistry}

540 Immunohistochemistry was carried out as described previously $(69,70)$ on paraffin541 embedded placenta/decidua (collected at E17.5) using goat rabbit anti-PTHrP (1:1000, 542 R87, generated against PTHrP(1-14) (71). The observer was blinded to genotype and sex 543 of all samples during analysis. Placental/decidual samples were fixed overnight in $4 \%$ 544 paraformaldehyde at $4^{\circ} \mathrm{C}$, stored in $70 \%$ ethanol, and embedded in paraffin wax until 545 further analysis. Sections $(5 \mu \mathrm{m})$ were taken onto chrome alum-coated slides, dewaxed in 546 Histoclear (National Diagnostics, Atlanta, GA), and rehydrated in graded ethanols. 547 Endogenous peroxidase was blocked for 30 min in $2 \% \mathrm{H}_{2} \mathrm{O}_{2}$ in methanol. After rinsing 548 with $0.05 \mathrm{M}$ phosphate buffered saline (PBS), samples were blocked with TNB 549 (Renaissance TSA indirect (Tyramide Signal Amplification) PerkinElmer Life Sciences cat 550 no- NEL700) for 60 min. PTHrP antibody (made against PTHrP (1-14) in house R87) $551 \quad 1: 1000$ or rabbit IgG (negative control) was applied for $2 \mathrm{hr}$ at room temperature in a 552 humid chamber. A secondary antibody (swine anti-rabbit, Dako) was applied for $30 \mathrm{~min}$ 553 at 1:300, followed by streptavidin horseradish peroxidase (Dako) 1:300 in the same 554 blocking solution for $30 \mathrm{~min}$. PTHrP staining was visualized with diaminobenzidine kit 555 (Dako) and conterstained with Mayer's hematoxylin. Samples were rinsed in PBS 556 between each step. PTHrP-DAB positive regions within the decidua and 557 spongiotrophoblast zones were quantified with MetaMorph® image analysis software 558 (Molecular Devices, San Jose, CA). The decidua and spongiotrophoblast zones were 559 manually defined. Colour thresholding was applied and compared to negative controls. 560 Integrated morphometry analysis was used to quantify DAB intensity, and frequency 561 parameters.

\section{$562 \quad$ Statistical analysis}

563 Statistically significant differences were determined by one-way or two-way ANOVA 564 followed by Sidak's post-hoc test or Fishers LSD posthoc test (uncorrected). Student's t- 
bioRxiv preprint doi: https://doi.org/10.1101/2020.06.09.141812; this version posted June 11, 2020. The copyright holder for this preprint (which was not certified by peer review) is the author/funder, who has granted bioRxiv a license to display the preprint in perpetuity. It is made available under aCC-BY 4.0 International license.

test was used where only one comparison was being made. To analyse PTHrP positive

566 areas in decidua/placentae, area under the curves were compared by Student's t-test.

567 


\section{Acknowledgments}

569 The authors thank the staff of the St. Vincent's Health Bioresources Centre for excellent

570 animal care and assistance. This work was partially supported by National Health and

571 Medical Research Council (Australia) Project Grants to N.A.S. and T.J.M. N.A. was

572 supported by The University of Melbourne International Research Scholarship and a St.

573 Vincent's Institute top-up scholarship. T.I was supported by Travel Grants from Mochida

574 Memorial Foundation for Medical and Pharmacological Research and The Foundation for

575 Growth Science, Japan. N.A.S. is supported by a National Health and Medical Research

576 Council (Australia) Senior Research Fellowship, and was supported in 2018 by the SVI

577 Brenda Shanahan Fellowship. St. Vincent's Institute is supported by the Victorian

578 Government's Operational Infrastructure Support Programme. 
579 Table 1. Trabecular structure of L5 ( $5^{\text {th }}$ lumbar vertebrae) from 14 week old male 580 Dmp1Cre.Pthlhf/f(hom) and Dmp1Cre(hom) controls by microcomputed tomography. Values 581 are mean \pm SEM. $\mathrm{n}=9-10 /$ group; $^{*} \mathrm{p}<0.05$ vs Dmp1Cre $e^{\text {(hom) }}$ by Student's t-test.

\begin{tabular}{|c|c|c|}
\hline & Dmp1Cre $e^{(h o m)}$ & Dmp1Cre.Pthlhf/f(hom) \\
\hline Trabecular bone volume/TV (\%) & $17.84 \pm 0.89$ & $21.32 \pm 1.16^{*}$ \\
\hline Trabecular number $(/ \mathrm{mm})$ & $3.20 \pm 0.13$ & $3.70 \pm 0.15^{*}$ \\
\hline Trabecular thickness $(\mu \mathrm{m})$ & $55.6 \pm 1.3$ & $57.3 \pm 1.0$ \\
\hline Trabecular separation $(\mu \mathrm{m})$ & $203.5 \pm 4.9$ & $185.4 \pm 6.4^{*}$ \\
\hline
\end{tabular}

582 
Table 2 Histomorphometry of tibia and serum biochemical data of 14 week-old male Dmp1Cre.Pthlhf/f(hom) and Dmp1Cre(hom) controls. Histomorphometry was measured in the distal tibial metaphyseal secondary spongiosa. Values are mean \pm SEM. $n=7-10$ /group. Abbreviations: BV: bone volume; BS: bone surface; B. Pm: bone perimeter; N.Ob: osteoblast numbers; B.Pm: bone perimeter; N.Oc: osteoclast numbers; Oc.Pm: osteoclast perimeter; P1NP: Procollagen type $1 \mathrm{~N}$-terminal propeptide; CTX1: C-telopeptide of type 5901 collagen.

\begin{tabular}{|l|cc|}
\hline & Dmp1Cre(hom) & Dmp1Cre.Pthlh/f(hom) \\
\hline Osteoid surface/BS (\%) & $6.16 \pm 1.85$ & $6.47 \pm 1.83$ \\
Osteoid thickness ( $\mu \mathrm{m})$ & $0.90 \pm 0.11$ & $1.20 \pm 0.23$ \\
Osteoblast surface/BS (\%) & $7.83 \pm 2.46$ & $9.02 \pm 2.55$ \\
Osteoblast numbers (N.Ob/B.Pm) (N/mm) & $6.72 \pm 2.09$ & $7.77 \pm 2.16$ \\
Osteoclast surface/BS (\%) & $3.77 \pm 0.38$ & $3.86 \pm 0.44$ \\
Osteoclast numbers (N.Oc/B.Pm) (N/mm) & $1.79 \pm 0.29$ & $1.93 \pm 0.23$ \\
N.Oc/Oc.Pm (N/mm) & $46.60 \pm 6.18$ & $49.79 \pm 1.50$ \\
Single-labeled mineralizing surface (sL.S/BS) & $22.4 \pm 2.4$ & $20.8 \pm 3.8$ \\
(\%) & & \\
Double-labeled mineralizing $\quad$ surface & $12.9 \pm 2.9$ & $15.9 \pm 4.2$ \\
(dL.S/BS) (\%) & $24.2 \pm 2.0$ & $26.3 \pm 3.3$ \\
Mineralising surface/BS (\%) & $1.09 \pm 0.08$ & $1.05 \pm 0.15$ \\
Mineral apposition rate ( $\mu$ /day) & $1.30 \pm 0.10$ & $1.41 \pm 0.32$ \\
Bone formation rate/BV (\%/day) & $29.6 \pm 2.3$ & $36.6 \pm 4.1$ \\
\hline P1NP (ng/ml) & $25.4 \pm 1.8$ & $21.5 \pm 1.6$ \\
CTX1 (ng/ml) & & \\
\hline
\end{tabular}


Table 3. Additional strength data from three point bending test on femora from 14 and 26 week old Dmp1Cre.Pthlhf/f(hom) (f/f) and $\operatorname{Dmp} 1 \mathrm{Cre}^{(\mathrm{hom})}(\mathrm{w} / \mathrm{w})$ mice. Values are mean \pm SEM. $\mathrm{n}=9-10 /$ group; ${ }^{*} \mathrm{p}<0.05$, ${ }^{* *} \mathrm{p}<0.01$, and ${ }^{* * *} \mathrm{p}<0.001$ vs sex- and age-matched w/w by Student's t-test (14 weeks old) and two-way ANOVA (26 weeks old) with uncorrected Fishers LSD post-hoc test.

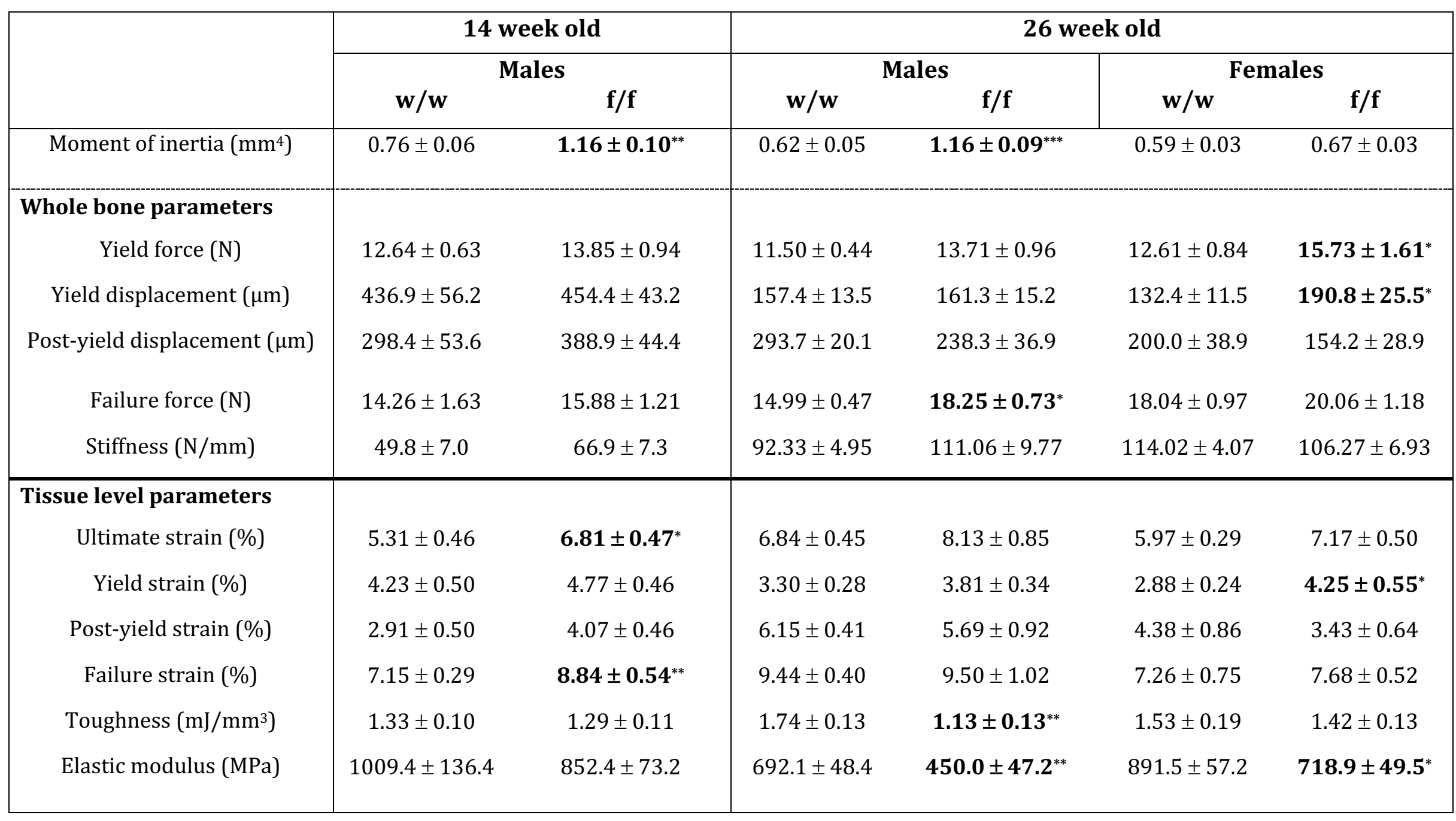


Table 4. Trabecular bone structure of 12 day old Dmp1Cre.Pthlhfff(hom) and Dmp1Cre(hom) mice. Trabecular bone was measured by histomorphometry in male and female tibiae. Data is shown as mean \pm SEM.

\begin{tabular}{|c|c|c|c|c|}
\hline & \multicolumn{2}{|c|}{ Male } & \multicolumn{2}{|r|}{ Female } \\
\hline & Dmp1Cre $e^{(\text {hom })}(\mathrm{n}=6)$ & $\begin{array}{c}\text { Dmp1Cre.Pthlhf/f } \\
\text { (hom) } \\
(\mathrm{n}=4)\end{array}$ & $\begin{array}{c}\text { Dmp1Cre } \\
(\mathrm{n}=4)\end{array}$ & Dmp1Cre.Pthlhf/f(hom) $(\mathrm{n}=3)$ \\
\hline Trabecular bone volume (\%) & $3.65 \pm 1.00$ & $5.19 \pm 2.11$ & $2.78 \pm 0.98$ & $8.38 \pm 4.70$ \\
\hline Trabecular number $(/ \mathrm{mm})$ & $1.36 \pm 0.30$ & $1.84 \pm 0.44$ & $1.12 \pm 0.32$ & $2.51 \pm 0.92$ \\
\hline Trabecular thickness $(\mu \mathrm{m})$ & $24.85 \pm 2.38$ & $25.63 \pm 3.78$ & $24.13 \pm 2.27$ & $29.15 \pm 5.97$ \\
\hline Trabecular separation $(\mu \mathrm{m})$ & $1101.06 \pm 407.40$ & $589.18 \pm 103.76$ & $1344.61 \pm 607.52$ & $476.28 \pm 155.84$ \\
\hline
\end{tabular}


Table 5. Biochemical analysis of milk samples. PTHrP content was measured by radioimmunoassay (RIA) in milk samples from mothers of Dmp1Cre.Pthlhfff(hom) and Dmp1Cre (hom) mice on day 12 of lactation. PTHrP equivalent concentration was measured by measuring cAMP response to milk treatment of UMR106-01 cells (bioassay). Data shown as mean \pm SEM. $n=7$ /group. No significant differences relating to genotype were detected by Student's t-test.

\begin{tabular}{|l|cc|}
\hline Mothers of: & Dmp1Cre & Dmp1Cre.Pthlhf/f(hom) \\
\hline PTHrP (pmol)/milk protein (g) by RIA & $64.54 \pm 13.53$ & $45.53 \pm 5.12$ \\
PTHrP (pmol)/milk protein (g) by & $581.68 \pm$ & $582.80 \pm 90.97$ \\
bioassay & 43.57 & \\
Milk protein (g/L) & $100.42 \pm 4.16$ & $99.28 \pm 7.61$ \\
\hline
\end{tabular}




\section{References}

607

608 1. Jepsen KJ, Silva MJ, Vashishth D, Guo XE, van der Meulen MC. Establishing

609 biomechanical mechanisms in mouse models: practical guidelines for systematically

610 evaluating phenotypic changes in the diaphyses of long bones. J Bone Miner Res.

$6112015 ; 30(6): 951-66$.

612 2. Abad V, Meyers JL, Weise M, Gafni RI, Barnes KM, Nilsson O, et al. The role of the 613 resting zone in growth plate chondrogenesis. Endocrinology. 2002;143(5):1851-7.

614 3. Pazzaglia UE, Beluffi G, Benetti A, Bondioni MP, Zarattini G. A review of the actual

615 knowledge of the processes governing growth and development of long bones. Fetal and 616 pediatric pathology. 2011;30(3):199-208.

617 4. Martin TJ. Parathyroid Hormone-Related Protein, Its Regulation of Cartilage and

618 Bone Development, and Role in Treating Bone Diseases. Physiol Rev. 2016;96(3):831-71.

619 5. Karaplis AC, Luz A, Glowacki J, Bronson RT, Tybulewicz VL, Kronenberg HM, et

620 al. Lethal skeletal dysplasia from targeted disruption of the parathyroid hormone-related

621 peptide gene. Genes \& Development. 1994;8(3):277-89.

622 6. Miao D, He B, Jiang Y, Kobayashi T, Soroceanu MA, Zhao J, et al. Osteoblast-

623 derived PTHrP is a potent endogenous bone anabolic agent that modifies the therapeutic

624 efficacy of administered PTH 1-34. J Clin Invest. 2005;115(9):2402-11.

625 7. Ansari N, Ho PW, Crimeen-Irwin B, Poulton IJ, Brunt AR, Forwood MR, et al.

626 Autocrine and Paracrine Regulation of the Murine Skeleton by Osteocyte-Derived

627 Parathyroid Hormone-Related Protein. J Bone Miner Res. 2018;33(1):137-53.

628 8. Standal T, Johnson RW, McGregor NE, Poulton IJ, Ho PW, Martin TJ, et al. gp130 in

629 late osteoblasts and osteocytes is required for PTH-induced osteoblast differentiation. J

630 Endocrinol. 2014;223(2):181-90.

631 9. Cho DC, Brennan HJ, Johnson RW, Poulton IJ, Gooi JH, Tonkin BA, et al. Bone

632 corticalization requires local SOCS3 activity and is promoted by androgen action via

633 interleukin-6. Nat Commun. 2017;8(1):806.

634 10. Mamillapalli R, VanHouten J, Dann P, Bikle D, Chang W, Brown E, et al. Mammary-

635 specific ablation of the calcium-sensing receptor during lactation alters maternal calcium

636 metabolism, milk calcium transport, and neonatal calcium accrual. Endocrinology.

637 2013;154(9):3031-42.

638 11. Karperien M, Lanser P, de Laat SW, Boonstra J, Defize LH. Parathyroid hormone

639 related peptide mRNA expression during murine postimplantation development: evidence for

640 involvement in multiple differentiation processes. The International journal of developmental

641 biology. 1996;40(3):599-608.

642 12. Kovacs CS, Lanske B, Hunzelman JL, Guo J, Karaplis AC, Kronenberg HM.

643 Parathyroid hormone-related peptide (PTHrP) regulates fetal-placental calcium transport

644 through a receptor distinct from the PTH/PTHrP receptor. Proc Natl Acad Sci U S A.

645 1996;93(26):15233-8.

646 13. Ferguson JE, 2nd, Gorman JV, Bruns DE, Weir EC, Burtis WJ, Martin TJ, et al.

647 Abundant expression of parathyroid hormone-related protein in human amnion and its

648 association with labor. Proceedings of the National Academy of Sciences of the United States

649 of America. 1992;89(17):8384-8.

650 14. Williams ED, Leaver DD, Danks JA, Moseley JM, Martin TJ. Effect of parathyroid hormone-related protein (PTHrP) on the contractility of the myometrium and localization of PTHrP in the uterus of pregnant rats. J Reprod Fertil. 1994;102(1):209-14. 
15. Meziani F, Van Overloop B, Schneider F, Gairard A. Parathyroid hormone-related protein-induced relaxation of rat uterine arteries: influence of the endothelium during gestation. J Soc Gynecol Investig. 2005;12(1):14-9.

16. Ramathal CY, Bagchi IC, Taylor RN, Bagchi MK. Endometrial decidualization: of mice and men. Semin Reprod Med. 2010;28(1):17-26.

17. Okada H, Tsuzuki T, Murata H. Decidualization of the human endometrium. Reprod Med Biol. 2018;17(3):220-7.

18. Sherafat-Kazemzadeh R, Schroeder JK, Kessler CA, Handwerger S. Parathyroid hormone-like hormone (PTHLH) represses decidualization of human uterine fibroblast cells by an autocrine/paracrine mechanism. The Journal of clinical endocrinology and metabolism. 2011;96(2):509-14.

19. Williams ED, Major BJ, Martin TJ, Moseley JM, Leaver DD. Effect of antagonism of the parathyroid hormone (PTH)/PTH-related protein receptor on decidualization in rat uterus. J Reprod Fertil. 1998;112(1):59-67.

20. Katoh N, Kuroda K, Tomikawa J, Ogata-Kawata H, Ozaki R, Ochiai A, et al. Reciprocal changes of $\mathrm{H} 3 \mathrm{~K} 27 \mathrm{ac}$ and $\mathrm{H} 3 \mathrm{~K} 27 \mathrm{me} 3$ at the promoter regions of the critical genes for endometrial decidualization. Epigenomics. 2018;10(9):1243-57. 21. Lim J, Burclaff J, He G, Mills JC, Long F. Unintended targeting of. Bone Res. 2017;5:16049.

22. San Martin S, Fitzgerald JS, Weber M, Párraga M, Sáez T, Zorn TM, et al. Stat3 and Socs 3 expression patterns during murine placenta development. Eur J Histochem. 2013;57(2):e19-e.

23. Ni H, Ding N-Z, Harper MJK, Yang Z-M. Expression of leukemia inhibitory factor receptor and gp130 in mouse uterus during early pregnancy. Molecular reproduction and development. 2002;63(2):143-50.

24. Johnson RW, Brennan HJ, Vrahnas C, Poulton IJ, McGregor NE, Standal T, et al. The primary function of gp130 signaling in osteoblasts is to maintain bone formation and strength, rather than promote osteoclast formation. J Bone Miner Res. 2014;29(6):1492-505. 25. Murphy VE, Smith R, Giles WB, Clifton VL. Endocrine regulation of human fetal growth: the role of the mother, placenta, and fetus. Endocrine reviews. 2006;27(2):141-69. 26. Holroyd CR, Osmond C, Barker DJ, Ring SM, Lawlor DA, Tobias JH, et al. Placental Size Is Associated Differentially With Postnatal Bone Size and Density. J Bone Miner Res. 2016;31(10):1855-64.

27. Smith FG, Jr., Alexander DP, Buckle RM, Britton HG, Nixon DA. Parathyroid hormone in foetal and adult sheep: the effect of hypocalcaemia. The Journal of endocrinology. 1972;53(3):339-48.

28. Brown EM, Gamba G, Riccardi D, Lombardi M, Butters R, Kifor O, et al. Cloning and characterization of an extracellular $\mathrm{Ca}(2+)$-sensing receptor from bovine parathyroid. Nature. 1993;366(6455):575-80.

29. Miao D, He B, Karaplis AC, Goltzman D. Parathyroid hormone is essential for normal fetal bone formation. J Clin Invest. 2002;109(9):1173-82.

30. Lanske B, Karaplis AC, Lee K, Luz A, Vortkamp A, Pirro A, et al. PTH/PTHrP receptor in early development and Indian hedgehog-regulated bone growth. Science. 1996;273(5275):663-6.

31. Kronenberg HM. PTHrP and skeletal development. Annals of the New York Academy of Sciences. 2006;1068:1-13.

32. Frost HM. Mechanical determinants of bone modeling. Metab Bone Dis Relat Res. 1982;4(4):217-29. 
33. Frost HM. The mechanostat: a proposed pathogenic mechanism of osteoporoses and the bone mass effects of mechanical and nonmechanical agents. Bone Miner. 1987;2(2):7385 .

34. Rauch F. Bone growth in length and width: the Yin and Yang of bone stability. $\mathrm{J}$ Musculoskelet Neuronal Interact. 2005;5(3):194-201.

35. Volkman SK, Galecki AT, Burke DT, Paczas MR, Moalli MR, Miller RA, et al. Quantitative trait loci for femoral size and shape in a genetically heterogeneous mouse population. Journal of bone and mineral research : the official journal of the American Society for Bone and Mineral Research. 2003;18(8):1497-505.

36. Aiken CE, Ozanne SE. Sex differences in developmental programming models. Reproduction. 2013;145(1):R1-13.

37. Oyster N. Sex differences in cancellous and cortical bone strength, bone mineral content and bone density. Age Ageing. 1992;21(5):353-6.

38. Nelson DA, Megyesi MS. Sex and ethnic differences in bone architecture. Current osteoporosis reports. 2004;2(2):65-9.

39. Gabel L, Macdonald HM, McKay HA. Sex Differences and Growth-Related Adaptations in Bone Microarchitecture, Geometry, Density, and Strength From Childhood to Early Adulthood: A Mixed Longitudinal HR-pQCT Study. Journal of bone and mineral research : the official journal of the American Society for Bone and Mineral Research. 2017;32(2):250-63.

40. Evans RK, Negus C, Antczak AJ, Yanovich R, Israeli E, Moran DS. Sex differences in parameters of bone strength in new recruits: beyond bone density. Med Sci Sports Exerc. 2008;40(11 Suppl):S645-53.

41. Eckstein F, Matsuura M, Kuhn V, Priemel M, Muller R, Link TM, et al. Sex differences of human trabecular bone microstructure in aging are site-dependent. J Bone Miner Res. 2007;22(6):817-24.

42. Beck TJ, Ruff CB, Scott WW, Jr., Plato CC, Tobin JD, Quan CA. Sex differences in geometry of the femoral neck with aging: a structural analysis of bone mineral data. Calcified tissue international. 1992;50(1):24-9.

43. Amin S, Khosla S. Sex- and age-related differences in bone microarchitecture in men relative to women assessed by high-resolution peripheral quantitative computed tomography. J Osteoporos. 2012;2012:129760.

44. Sigurdsson G, Aspelund T, Chang M, Jonsdottir B, Sigurdsson S, Eiriksdottir G, et al. Increasing sex difference in bone strength in old age: The Age, Gene/Environment Susceptibility-Reykjavik study (AGES-REYKJAVIK). Bone. 2006;39(3):644-51.

45. Ackerman A, Thornton JC, Wang J, Pierson RN, Jr., Horlick M. Sex difference in the effect of puberty on the relationship between fat mass and bone mass in 926 healthy subjects, 6 to 18 years old. Obesity (Silver Spring). 2006;14(5):819-25.

46. van der Eerden BC, Emons J, Ahmed S, van Essen HW, Lowik CW, Wit JM, et al. Evidence for genomic and nongenomic actions of estrogen in growth plate regulation in female and male rats at the onset of sexual maturation. The Journal of endocrinology. 2002;175(2):277-88.

47. Ren SG, Malozowski S, Sanchez P, Sweet DE, Loriaux DL, Cassorla F. Direct administration of testosterone increases rat tibial epiphyseal growth plate width. Acta Endocrinol (Copenh). 1989;121(3):401-5.

48. Sims NA, Brennan K, Spaliviero J, Handelsman DJ, Seibel MJ. Perinatal testosterone surge is required for normal adult bone size but not for normal bone remodeling. Am J Physiol Endocrinol Metab. 2006;290(3):E456-62. 
49. Ye L, Mishina Y, Chen D, Huang H, Dallas SL, Dallas MR, et al. Dmp1-deficient mice display severe defects in cartilage formation responsible for a chondrodysplasia-like phenotype. The Journal of biological chemistry. 2005;280(7):6197-203.

50. Miao D, Su H, He B, Gao J, Xia Q, Zhu M, et al. Severe growth retardation and early lethality in mice lacking the nuclear localization sequence and C-terminus of PTH-related protein. Proc Natl Acad Sci U S A. 2008;105(51):20309-14.

51. Bikle D, Majumdar S, Laib A, Powell-Braxton L, Rosen C, Beamer W, et al. The skeletal structure of insulin-like growth factor I-deficient mice. Journal of bone and mineral research : the official journal of the American Society for Bone and Mineral Research. 2001;16(12):2320-9.

52. Liu JP, Baker J, Perkins AS, Robertson EJ, Efstratiadis A. Mice carrying null mutations of the genes encoding insulin-like growth factor I (Igf-1) and type 1 IGF receptor (Igf1r). Cell. 1993;75(1):59-72.

53. Vashishth D. Small animal bone biomechanics. Bone. 2008;43(5):794-7.

54. Ferrari SL, Rizzoli R, Bonjour JP. Parathyroid hormone-related protein production by primary cultures of mammary epithelial cells. Journal of cellular physiology. 1992;150(2):304-11.

55. Hyttinen JM, Korhonen VP, Hiltunen MO, Myohanen S, Janne J. High-level expression of bovine beta-lactoglobulin gene in transgenic mice. J Biotechnol. 1998;61(3):191-8.

56. VanHouten JN, Dann P, Stewart AF, Watson CJ, Pollak M, Karaplis AC, et al. Mammary-specific deletion of parathyroid hormone-related protein preserves bone mass during lactation. Journal of Clinical Investigation. 2003;112(9):1429-36. 57. Lu Y, Xie Y, Zhang S, Dusevich V, Bonewald LF, Feng JQ. DMP1-targeted Cre expression in odontoblasts and osteocytes. J Dent Res. 2007;86(4):320-5.

58. He B, Deckelbaum RA, Miao D, Lipman ML, Pollak M, Goltzman D, et al. Tissuespecific targeting of the pthrp gene: the generation of mice with floxed alleles. Endocrinology. 2001;142(5):2070-7.

59. Gillies BR, Ryan BA, Tonkin BA, Poulton IJ, Ma Y, Kirby BJ, et al. Absence of Calcitriol Causes Increased Lactational Bone Loss and Lower Milk Calcium but Does Not Impair Post-lactation Bone Recovery in Cyp27b1 Null Mice. J Bone Miner Res. 2018;33(1):16-26.

60. McFarlane L, Truong V, Palmer JS, Wilhelm D. Novel PCR assay for determining the genetic sex of mice. Sex Dev. 2013;7(4):207-11.

61. Takyar FM, Tonna S, Ho PW, Crimeen-Irwin B, Baker EK, Martin TJ, et al. EphrinB2/EphB4 inhibition in the osteoblast lineage modifies the anabolic response to parathyroid hormone. J Bone Miner Res. 2013;28(4):912-25.

62. Sims NA, Clement-Lacroix P, Da Ponte F, Bouali Y, Binart N, Moriggl R, et al. Bone homeostasis in growth hormone receptor-null mice is restored by IGF-I but independent of Stat5. J Clin Invest. 2000;106(9):1095-103.

63. Sims NA, Jenkins BJ, Nakamura A, Quinn JM, Li R, Gillespie MT, et al. Interleukin11 receptor signaling is required for normal bone remodeling. J Bone Miner Res. 2005;20(7):1093-102.

64. Williamson L, Hayes A, Hanson ED, Pivonka P, Sims NA, Gooi JH. High dose dietary vitamin D3 increases bone mass and strength in mice. Bone Rep. 2017;6:44-50. 65. Grill V, Ho P, Body JJ, Johanson N, Lee SC, Kukreja SC, et al. Parathyroid hormonerelated protein: elevated levels in both humoral hypercalcemia of malignancy and hypercalcemia complicating metastatic breast cancer. J Clin Endocrinol Metab. 1991;73(6):1309-15. 
798 66. Ho PW, Goradia A, Russell MR, Chalk AM, Milley KM, Baker EK, et al.

799 Knockdown of PTHR1 in osteosarcoma cells decreases invasion and growth and increases

800 tumor differentiation in vivo. Oncogene. 2015;34(22):2922-33.

801 67. Hammonds RG, Jr., McKay P, Winslow GA, Diefenbach-Jagger H, Grill V, Glatz J,

802 et al. Purification and characterization of recombinant human parathyroid hormone-related

803 protein. The Journal of biological chemistry. 1989;264(25):14806-11.

804 68. McLeod MJ. Differential staining of cartilage and bone in whole mouse fetuses by

805 alcian blue and alizarin red S. Teratology. 1980;22(3):299-301.

806 69. McGregor NE, Poulton IJ, Walker EC, Pompolo S, Quinn JM, Martin TJ, et al.

807 Ciliary neurotrophic factor inhibits bone formation and plays a sex-specific role in bone

808 growth and remodeling. Calcif Tissue Int. 2010;86(3):261-70.

809 70. Walker EC, McGregor NE, Poulton IJ, Solano M, Pompolo S, Fernandes TJ, et al.

810 Oncostatin M promotes bone formation independently of resorption when signaling through

811 leukemia inhibitory factor receptor in mice. J Clin Invest. 2010;120(2):582-92.

812 71. Lam MH, Thomas RJ, Loveland KL, Schilders S, Gu M, Martin TJ, et al. Nuclear

813 transport of parathyroid hormone $(\mathrm{PTH})$-related protein is dependent on microtubules.

814 Molecular endocrinology. 2002;16(2):390-401. 
819 Figure 1 Supplement 1: No difference in trabecular structure or metaphyseal 820 diameter in femora from Dmp1Cre.Pthlhf/f(het) mice and littermate Dmp1Cre(het) controls. Trabecular structure of distal femoral primary spongiosa analysed by microCT in male and female mice at 16 and 26 weeks of age. Trabecular bone volume, trabecular number, trabecular thickness, trabecular separation, and metaphyseal periosteal perimeter are shown as mean (dot), interquartile range (box), median (line) and range; $n=9-10 /$ group. No significant differences associated with genotype were detected (two-way ANOVA). Y-axes are drawn to match those of Figure 1 to allow comparison with Dmp1Cre.Pthlhf/f(hom) mice. Breeding strategy is shown in Figure 1A. weeks of age. Anteroposterior (C) and mediolateral (D) width, measured by micro-CT at the midshaft. E-I: Femoral marrow area (E), cortical bone area (F), thickness (G), and both endocortical (H) and periosteal (I) perimeter were analysed in cortical ROI by micro-CT . Data are shown as mean (dot), interquartile range (box), median (line) and range; $\mathrm{n}=9-10$ /group. ${ }^{*} \mathrm{p}<0.05$, and ${ }^{* *} \mathrm{p}<0.01$ compared to sex- and age-matched Dmp1Cre (het) by two-way ANOVA. Y-axes are drawn to match those of Figure 2 to allow comparison with Dmp1Cre.Pthlhf/f(hom) mice. Breeding strategy is shown in Figure 1A. Dmp1Cre ${ }^{\text {(het) }}$ controls at 26 weeks of age. Shown are ultimate force (A), ultimate deformation (B), ultimate stress (C), yield stress (D), and failure stress (E). Data shown as mean (dot), interquartile range (box), median (line) and range, $n=9-10 /$ group. No significant differences relating to genotype were detected by two-way ANOVA.

\section{Figure 5 Supplement 1: Additional micro-CT data of E18.5 Dmp1Cre.Pthlhf/f(hom)} embryos. Bone area and tissue area were analysed in cortical ROI by micro-CT in three different regions of femora: $20 \%$ of the mineralized length distal to the proximal end of the mineralized region (Top 20\%), midshaft (Mid), and 20\% of the mineralized length proximal to the distal end of the mineralized region (Bottom 20\%); see Figure 5A. Data is shown as mean \pm SEM with individual data points, ${ }^{*} \mathrm{p}<0.05$ and ${ }^{* *} \mathrm{p}<0.01$ compared to controls by two-way ANOVA. mean polar moment of inertia $(\mathbf{D}, \mathbf{G})$, bone area $(\mathbf{I}, \mathbf{K})$, and cross sectional area $(\mathbf{J}, \mathbf{L})$ of 
857 region (Top 20\%), midshaft (Mid), and 20\% of the mineralized length proximal to the

858 distal end of the mineralized region (Bottom 20\%). Data is shown as mean \pm SEM with

859 individual data points. No significant differences relating to genotype were detected by

860 two-way ANOVA.

861 Figure 6 Supplement 1: Embryo weight, placental weight, and decidual PTHrP of

862 Dmp1Cre.Pthlhf/f(het) mice. Embryo weight (A), placental weight (B) and embryo to

863 placental weight ratio (C) of Dmp1Cre.Pthlhf/f(het) and Dmp1Cre ${ }^{(\text {het })}$ embryos at E17.5. (D)

864 Frequency of PTHrP stained objects segregated by staining intensity in the

865 spongiotrophoblast layer and decidua from placental/decidual samples from

866 Dmp1Cre.Pthlhf/f(het) and Dmp1Cre(het) embryos. (F,G) Quantitation of total decidual area

867 and spongiotrophoblast area; mean \pm SEM with individual data points. No significant

868 differences relating to genotype were detected by two-way ANOVA. 
Figure 1 Supplement 1

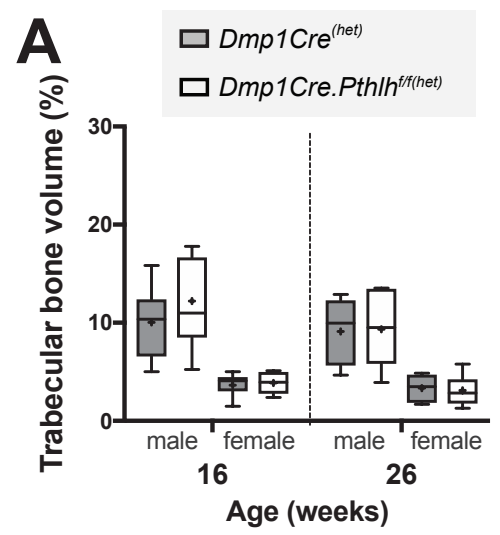

B

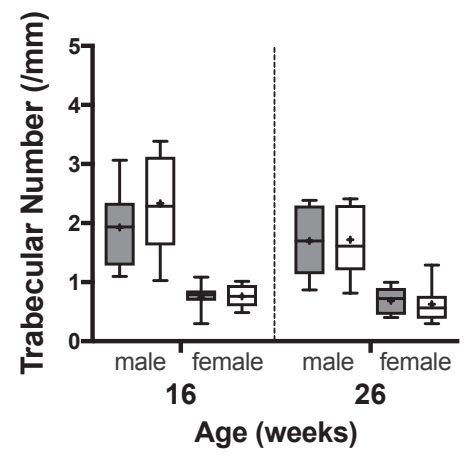

E

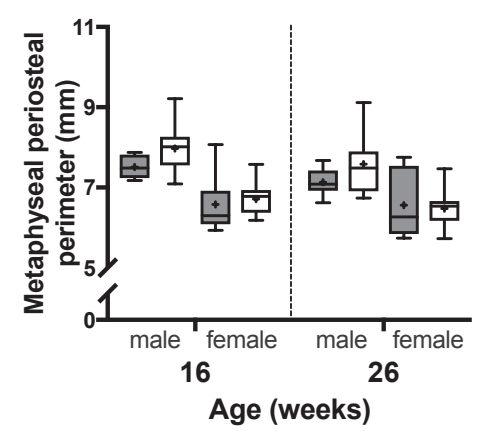

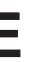

C

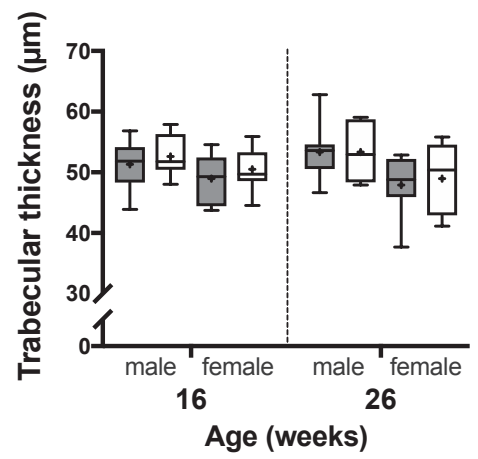

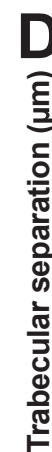

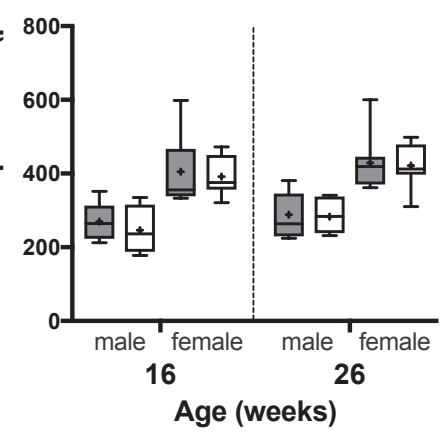


Figure 2 Supplement 1

A

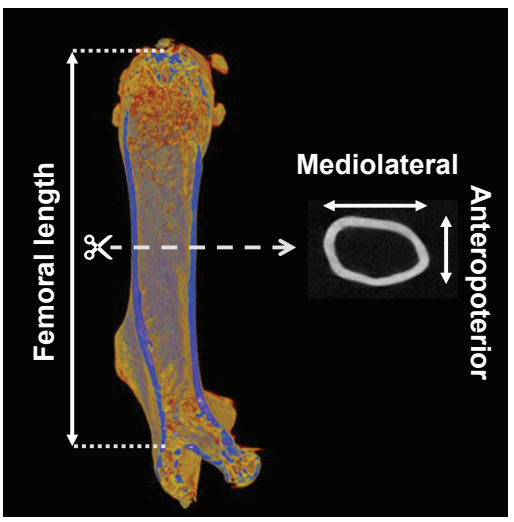

D

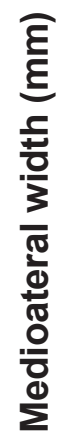

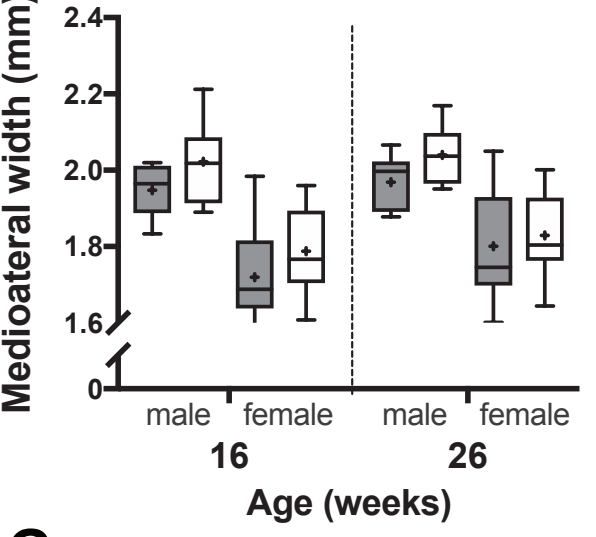

G

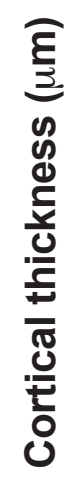

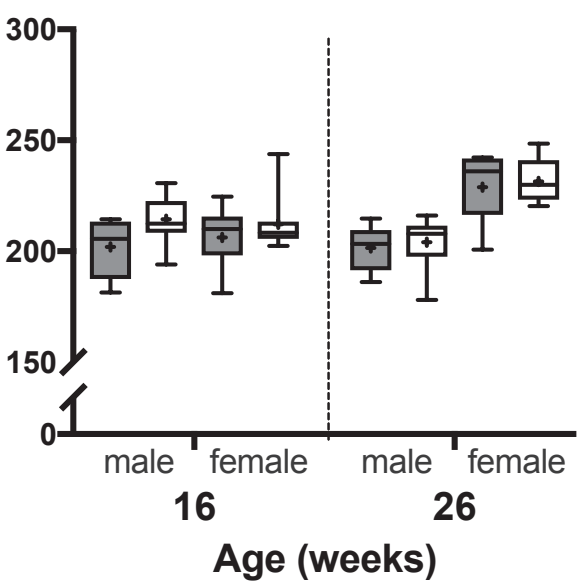

B
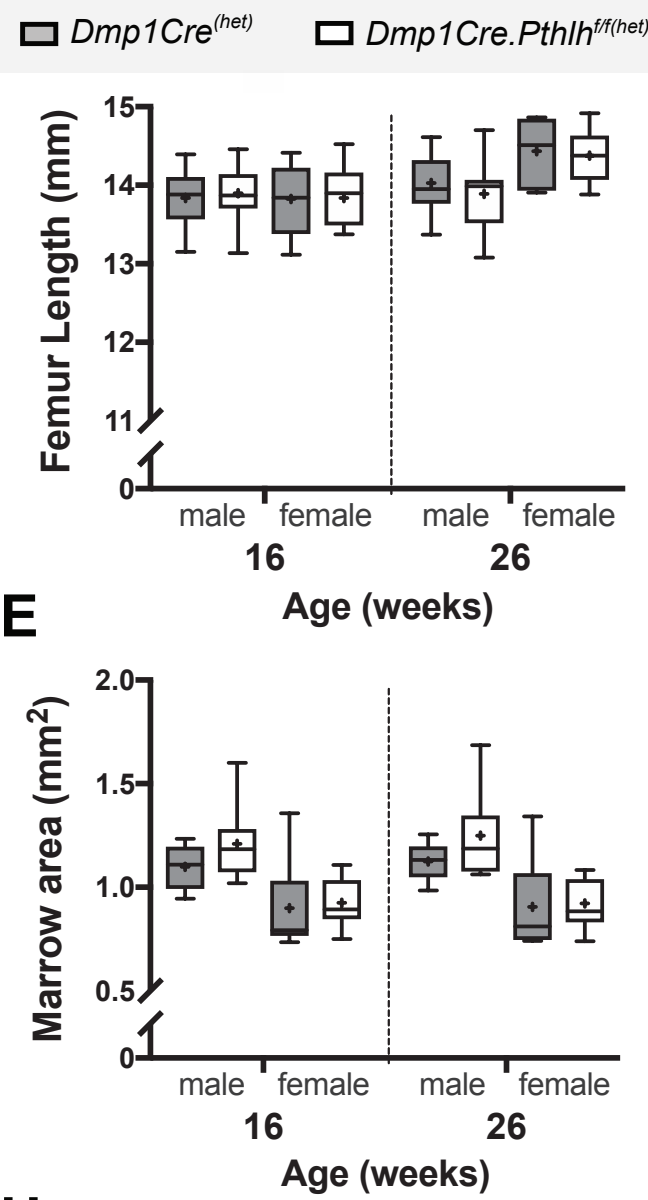

H

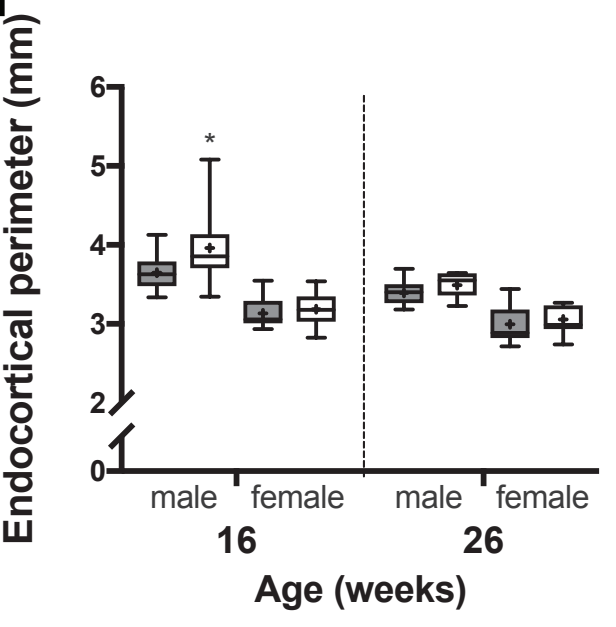

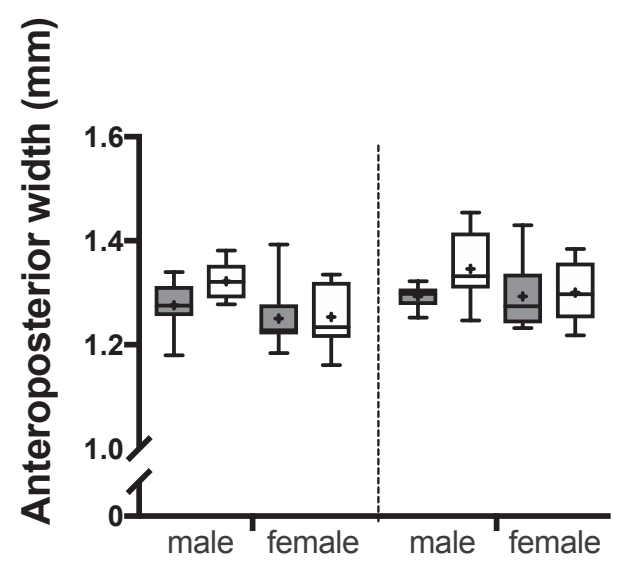

F
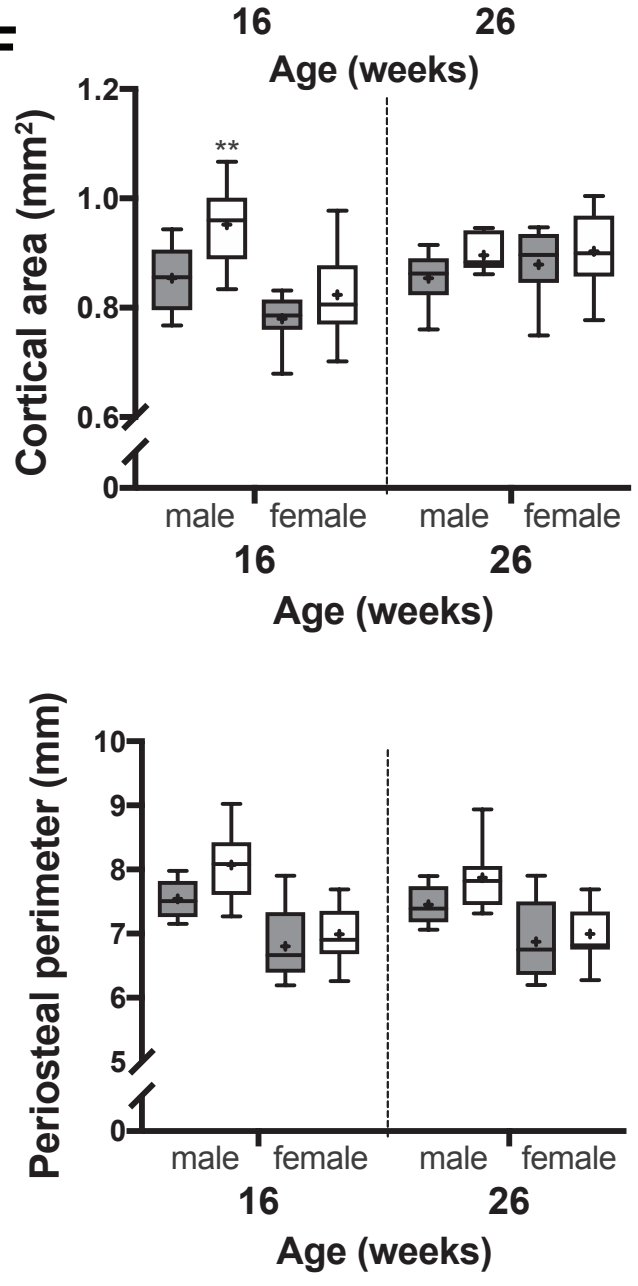
Figure 3 Supplement 1
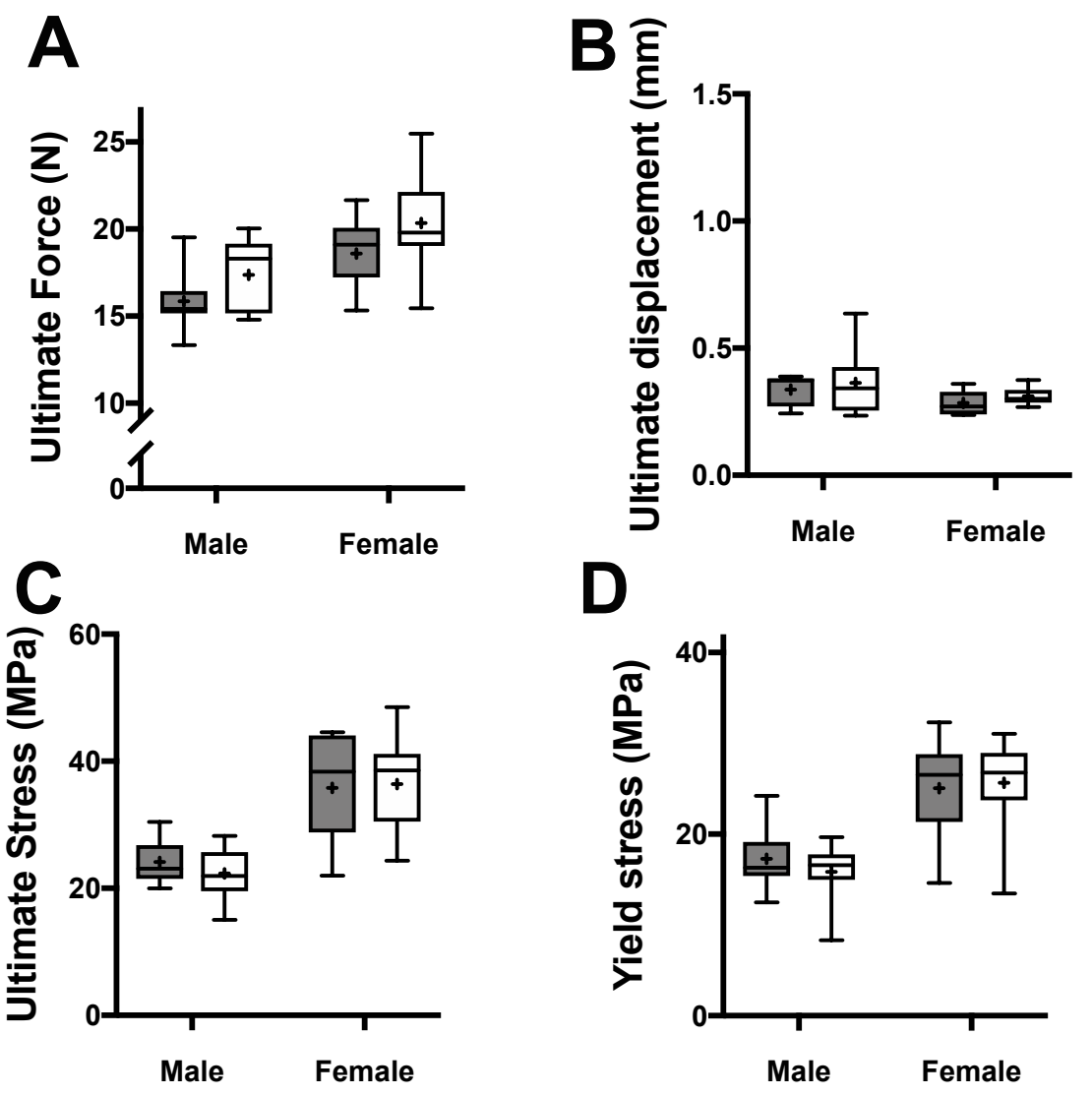

$\square$ Dmp1Cre ${ }^{(\text {het })}$

$\square$ Dmp1Cre.Pthlh ${ }^{f / f(h e t)}$
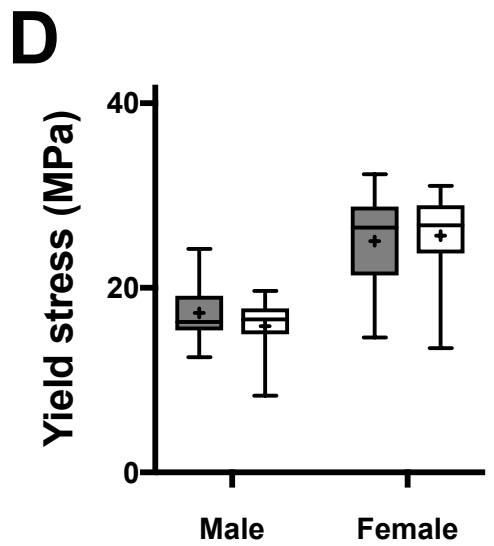
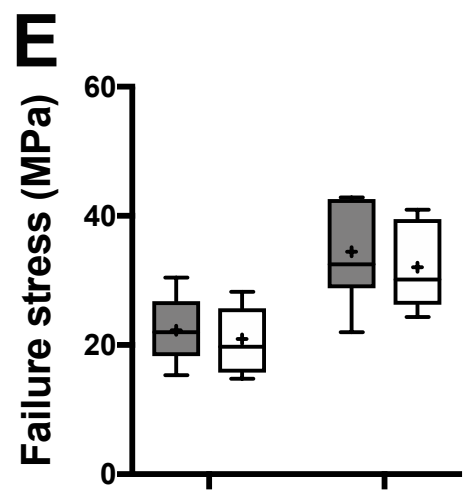

Male

Female 


\section{Figure 5 Supplement 1}

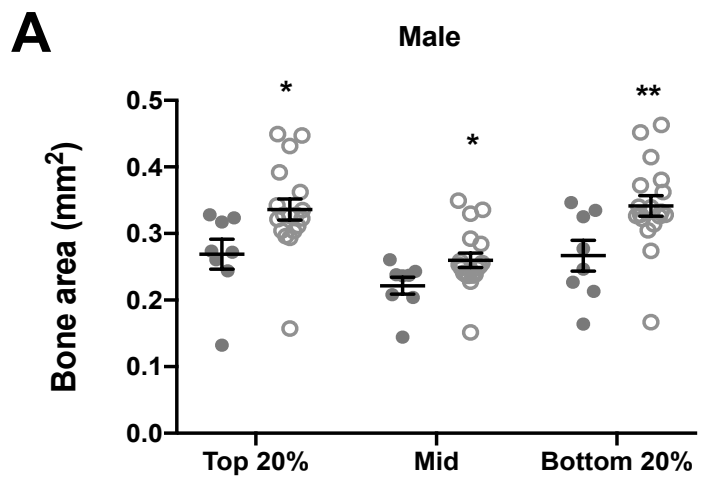

C

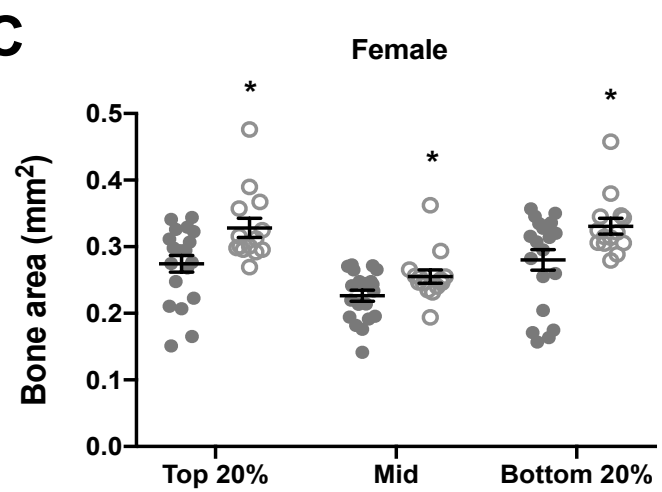

B

- Dmp1Cre ${ }^{(h o m)}$

- Dmp1Cre.Pthlh ${ }^{f / f(h o m)}$

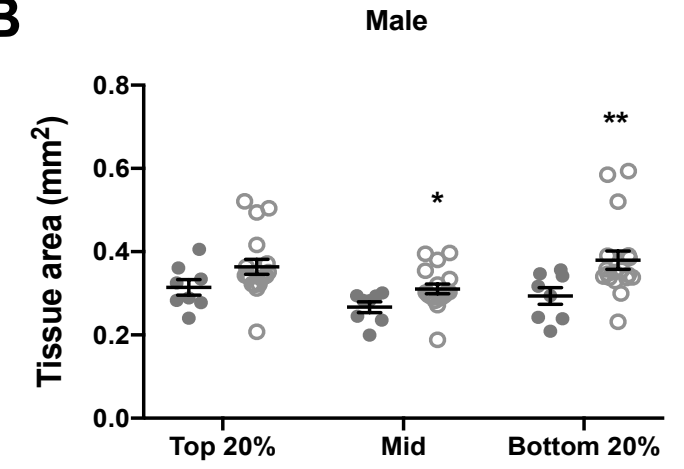

D

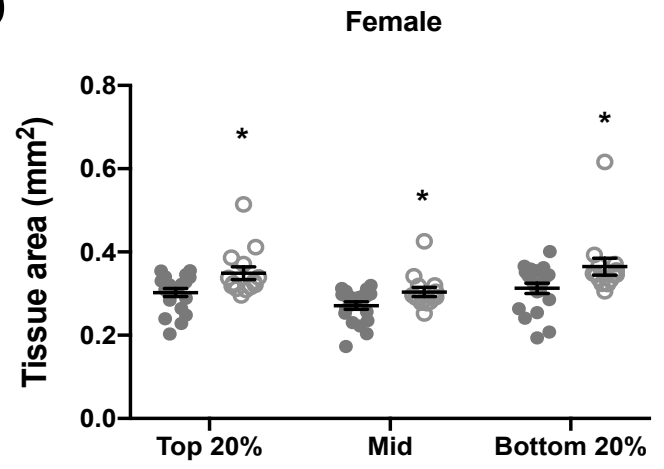




\section{Figure 5 Supplement 2}

A Dmp1Cre ${ }^{(\text {het })}$

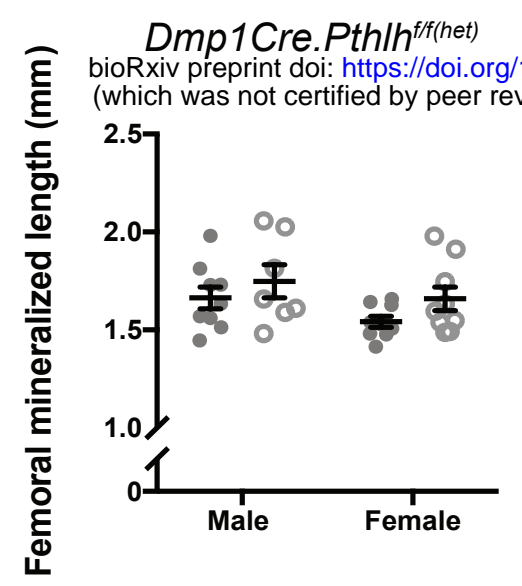

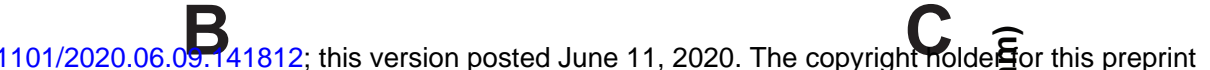
) is the author/funder, who bas granted bioRxiv Mlidense to display the preprint ingerpetyity. It is

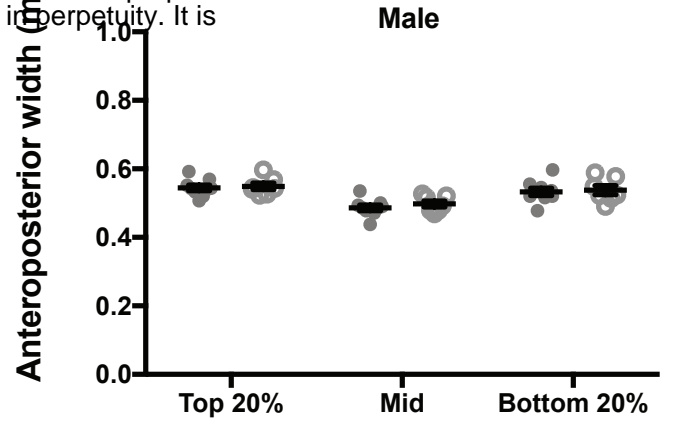

D

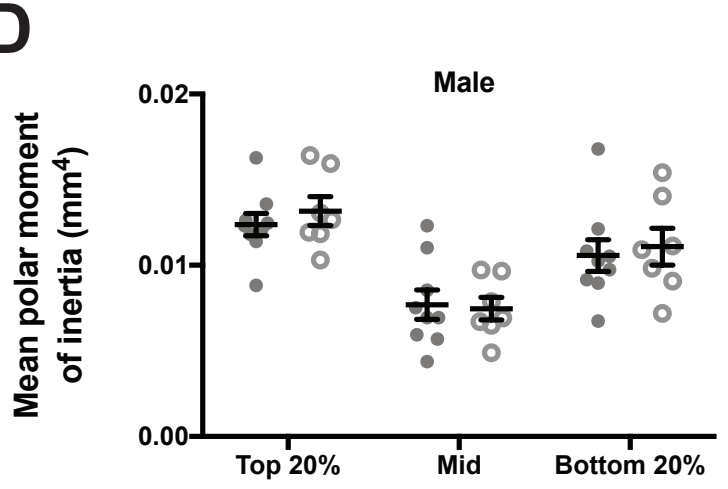

E
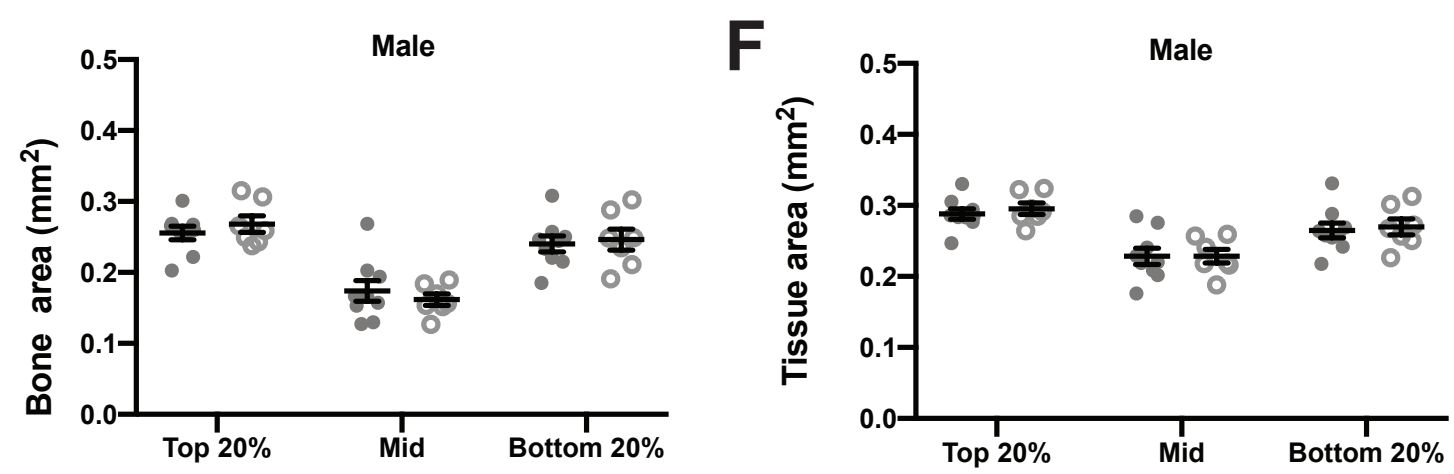

G

H
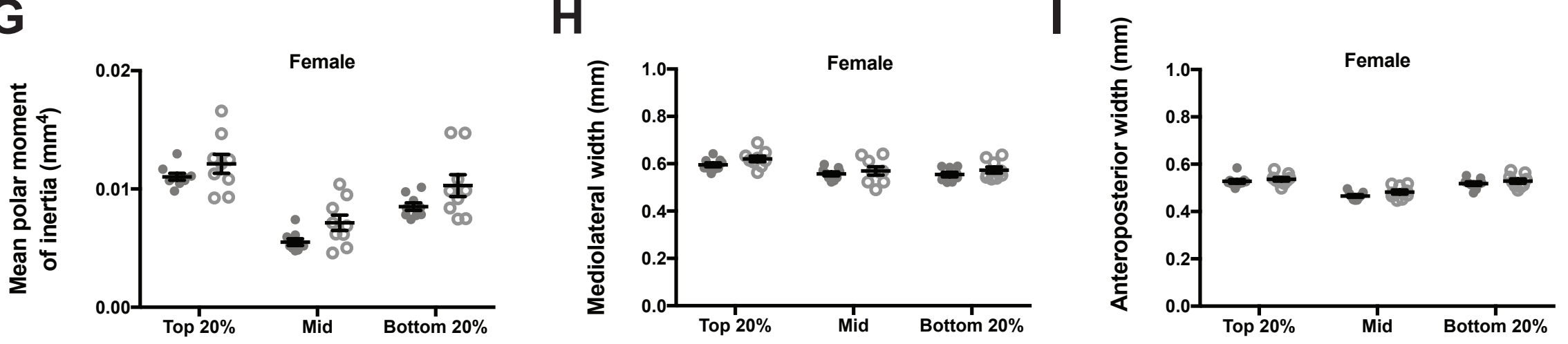

$\mathbf{J}$

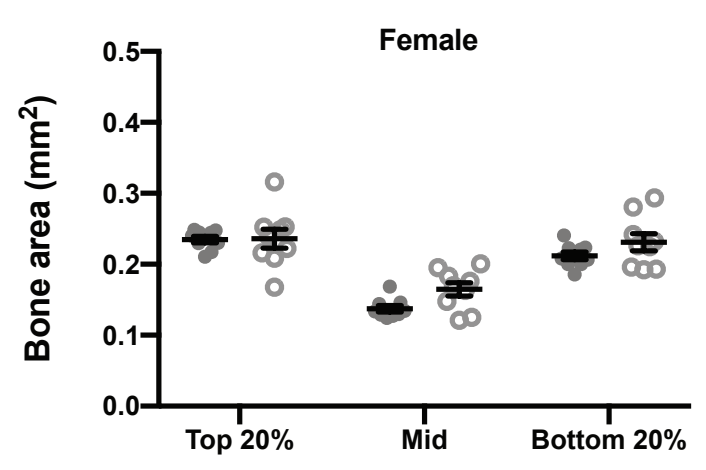

K

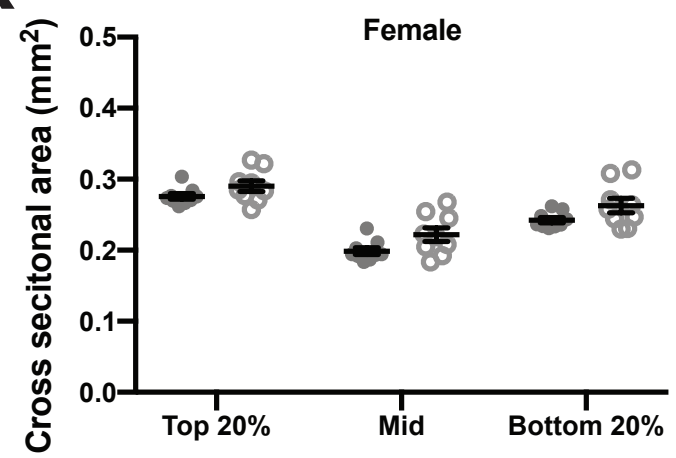




\section{Figure 6 Supplement 1}

A $\quad \circ$ Dmp1Cre ${ }^{\text {(het) }}$

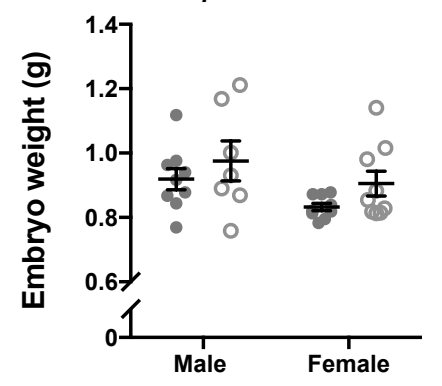

E

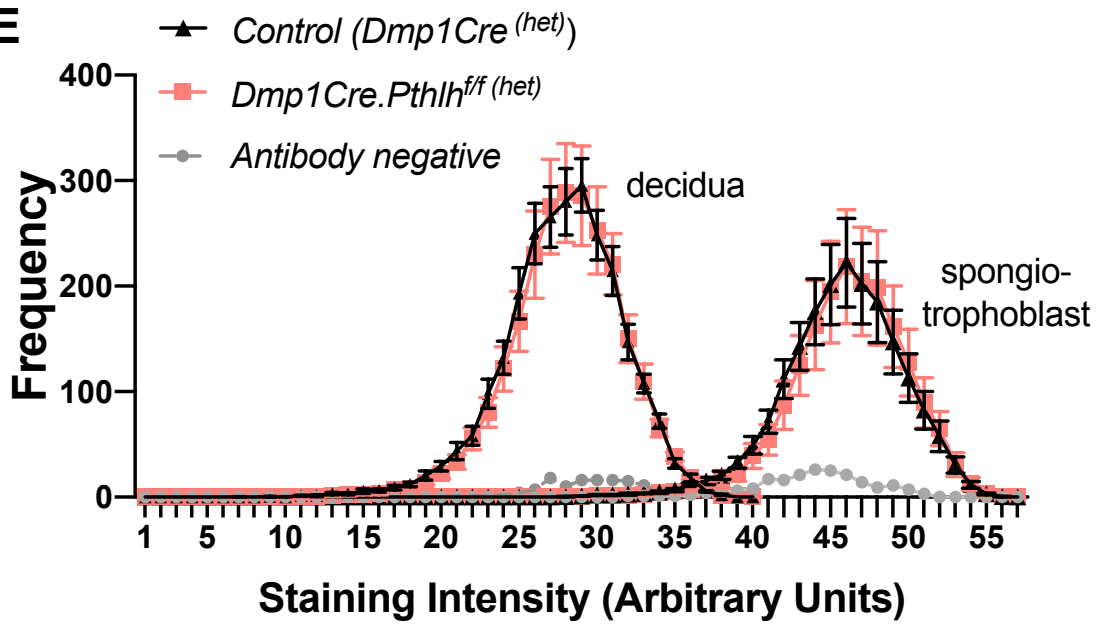

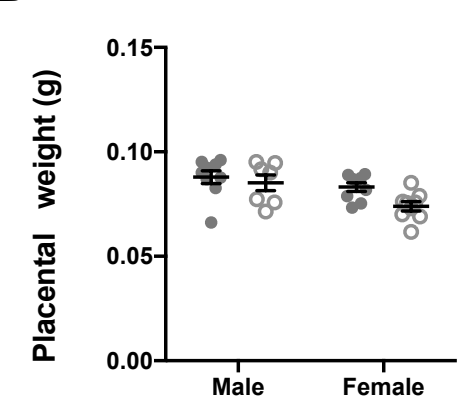

C

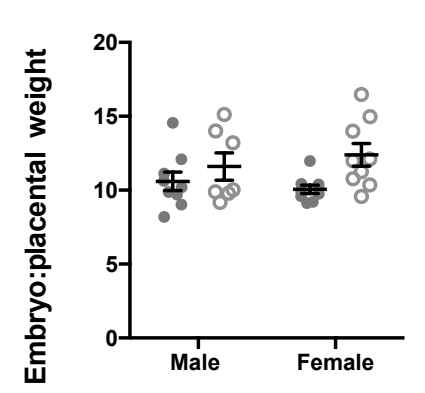

F

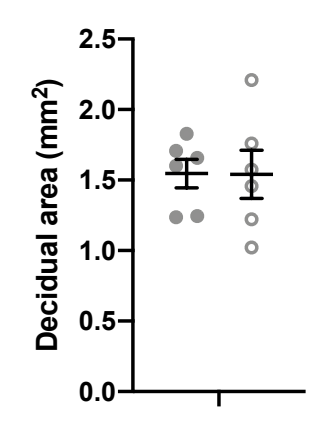

D

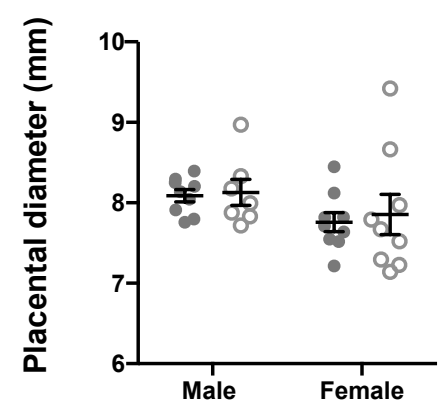

G

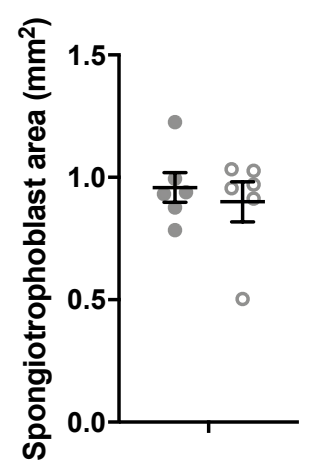

